Nova Southeastern University

Florida

NOVA SOUTHEASTERN

UNIVERSITY

NSUWorks

\title{
Characterization of Polar Organic Components in Fine Aerosols in the Southeastern United States: Identity, Origin, and Evolution
}

Song Gao

University of Arizona, sg1002@nova.edu

Jason D. Surratt

California Institute of Technology

Eladio M. Knipping

Electric Power Research Institute

Eric S. Edgerton

Atmospheric Research and Analysis, Inc.

Mona Shahgholi

California Institute of Technology

See next page for additional authors

Follow this and additional works at: https://nsuworks.nova.edu/cnso_chemphys_facarticles Part of the Environmental Chemistry Commons

\section{NSUWorks Citation}

Gao, S., Surratt, J. D., Knipping, E. M., Edgerton, E. S., Shahgholi, M., \& Seinfeld, J. H. (2006). Characterization of Polar Organic Components in Fine Aerosols in the Southeastern United States: Identity, Origin, and Evolution. Journal of Geophysical Research: Atmospheres, 111, (D14314), 1 - 27. https://doi.org/10.1029/2005JD006601. Retrieved from https://nsuworks.nova.edu/ cnso_chemphys_facarticles/154 


\section{Authors}

Song Gao, Jason D. Surratt, Eladio M. Knipping, Eric S. Edgerton, Mona Shahgholi, and John H. Seinfeld 


\title{
Characterization of polar organic components in fine aerosols in the southeastern United States: Identity, origin, and evolution
}

\author{
Song Gao, ${ }^{1}$ Jason D. Surratt, ${ }^{2}$ Eladio M. Knipping, ${ }^{3}$ Eric S. Edgerton, ${ }^{4}$ Mona Shahgholi, ${ }^{2}$ \\ and John H. Seinfeld ${ }^{1}$
}

Received 19 August 2005; revised 13 December 2005; accepted 28 February 2006; published 27 July 2006.

[1] Filter samples of fine aerosols collected in the Southeastern United States in June 2004 were analyzed for the characterization of polar organic components. Four analytical techniques, liquid chromatography -mass spectrometry, ion trap mass spectrometry, laser desorption ionization mass spectrometry, and high-resolution mass spectrometry, were used for identification and quantification. Forty distinct species were detected, comprising on average $7.2 \%$ and $1.1 \%$ of the total particulate organic mass at three inland sites and a coastal site, respectively. The relative abundance of these species displays a rather consistent distribution pattern in the inland region, whereas a different pattern is found at the coastal site. Chemical and correlation analyses suggest that the detected species are secondary in nature and originate from terpene oxidation, with possible participation of $\mathrm{NO}_{\mathrm{x}}$ and $\mathrm{SO}_{2}$. It is estimated that polar, acidic components in fine aerosols in the Southeastern United States cover a molecular weight range of 150-400 Da and do not appear to be oligomeric. Other components with MW up to 800 Da may also be present. The detected polar organic species are similar to humic-like substances (HULIS) commonly found in fine aerosols in other rural areas. We present the first, direct evidence that atmospheric processing of biogenic emissions can lead to the formation of certain HULIS species in fine aerosols, and that this may be a typical pathway in the background atmosphere in continental regions; nevertheless, a natural source for HULIS, such as from aquatic and/or terrestrial humic/fulvic acids and their degradation products, cannot be precluded.

Citation: Gao, S., J. D. Surratt, E. M. Knipping, E. S. Edgerton, M. Shahgholi, and J. H. Seinfeld (2006), Characterization of polar organic components in fine aerosols in the southeastern United States: Identity, origin, and evolution, J. Geophys. Res., 111, D14314, doi:10.1029/2005JD006601.

\section{Introduction}

[2] A subject of intense interest is the composition, sources and formation pathways of organic aerosols because of their role in climate forcing and their adverse effects on human health. The Southeastern United States (SE US) is of particular interest from a global point of view for its generally rural environment interspersed with a number of urban areas. Ample subtropical vegetation provides a constant source of biogenic volatile organic compounds (VOC), such as isoprene, monoterpenes and sesquiterpenes, in this large region. Also present are several urban areas, varying from small cities to metropolitan ones, and burgeoning industrial activities including coal-fired power plants, petroleum processing and maritime shipping, all contributing

\footnotetext{
${ }^{1}$ Departments of Environmental Science and Engineering and Chemical Engineering, California Institute of Technology, Pasadena, California, USA.

${ }^{2}$ Department of Chemistry, California Institute of Technology, Pasadena, California, USA.

${ }^{3}$ Electric Power Research Institute, Palo Alto, California, USA.

${ }^{4}$ Atmospheric Research and Analysis, Inc., Cary, North Carolina, USA.

Copyright 2006 by the American Geophysical Union. 0148-0227/06/2005JD006601
}

to background and heightened levels of $\mathrm{SO}_{2}, \mathrm{NO}_{\mathrm{x}}$ and anthropogenic VOC. Together with biogenic emissions, these emissions can contribute to the formation of ozone and particulate matter (PM). Prevailing winds can bring different source air masses to inland regions versus coastal areas. A number of field measurements have been carried out to study this region, including the Atlanta Supersite Experiment [e.g., Lim and Turpin, 2002] and the Southeastern Aerosol and Visibility Study (SEAVS) [e.g., Yu et al., 2005]. The Southeastern Aerosol Research and Characterization Study (SEARCH) was initiated in mid-1998 to carry out systematic measurements of temporal and spatial variability of $\mathrm{PM}$, in particular $\mathrm{PM}_{2.5}$ (i.e., aerosols with aerodynamic diameter less than $2.5 \mu \mathrm{m}$, also commonly called fine aerosols), gases relevant to secondary $\mathrm{O}_{3}$ formation, and surface meteorology. Among the myriad of measurements and analyses to date, the organic composition of $\mathrm{PM}_{2.5}$ has been explored, such as a source apportionment analysis by Zheng et al. [2002]. Zheng et al. concluded that the major source contributors to organic PM were wood combustion $(25-66 \%)$, diesel exhaust $(14-30 \%)$, meat cooking $(5-12 \%)$ and gasoline-powered motor vehicle exhaust $(0-10 \%)$. Altogether, the individual components identified and quantified by the gas chromatography-mass 
spectrometry (GC-MS) account for on average $12 \%$ of the total organic mass in $\mathrm{PM}_{2.5}$. An immediate question arises as to the identity of the nearly $90 \%$ of the $\mathrm{PM}_{2.5}$ organic mass. Since GC-MS is intrinsically amenable to identifying relatively nonpolar species (unless derivatization techniques are employed), and it is speculated that secondary organic aerosol (SOA) formation occurs more substantially in the summer, one central goal of this work is to understand the chemical nature, sources and evolution pathways of the relatively polar species in $\mathrm{PM}_{2.5}$ in the SE US.

[3] Answers to these questions, and indeed, more detailed knowledge of the organic composition of $\mathrm{PM}_{2.5}$ in the $\mathrm{SE}$ US, can have broader impacts on several fronts. Polar, more oxidized species may play important roles in determining the hygroscopicity and cloud condensation nuclei (CCN) activity of aerosols [Cruz and Pandis, 1998, 2000; Choi and Chan, 2002]. The detection of oligomers in SOA from recent chamber experiments, attributed tentatively to acid catalysis, poses the possibility that oligomers may be present in ambient aerosols [Kalberer et al., 2004; Tolocka et al., 2004; Gao et al., 2004a, 2004b; Baltensperger et al., 2005]. To date, no definitive detection of oligomers in ambient aerosols has been reported. There are limitations of laboratory chamber experiments, such as the insufficient aging time. The longest duration of SOA evolution was $\sim 30$ hours for Kalberer et al. [2004], which is less than that in the ambient air (e.g., about 1 week for $\mathrm{PM}_{2.5}$ in the SE US). Oxidation and even decomposition of oligomers may take place in the latter stage of aging. In addition, the hydrocarbon concentrations used in chamber experiments tend to be about an order of magnitude higher than those in the atmosphere, and synergistic effects of multiple reactants and oxidants generally have not been simulated yet in laboratory experiments. Theoretical calculations do not seem to support the stable presence of species formed via accretion reactions alone [Barsanti and Pankow, 2004]. Answers to whether oligomers are present in $\mathrm{PM}_{2.5}$ in the SE US and whether particle acidity plays a role in affecting aerosol composition are of great interest in bridging the gap between laboratory studies and ambient realities.

[4] In rural areas worldwide, a large number of organic species have been detected in aerosols, as well as fog and cloud water [e.g., Kavouras et al., 1999; Krivacsy et al., 2001; Pio et al., 2001; Kiss et al., 2003; Cappiello et al., 2003]. These species have been found to be generally rather polar and even water-soluble; however, their chemical identity remains elusive. The ensemble of these species is often referred to as humic-like substances (HULIS), and hypothesis has been made as to a direct link between HULIS species in atmospheric aerosols, with MW estimated to be on the order of several hundreds, and humic/fulvic acids naturally occurring in terrestrial and aquatic environments [Gelencser et al., 2002]. Interestingly, a secondary photochemical source for HULIS has never been precluded, although direct evidence for this pathway has been lacking. A better understanding of the $\mathrm{PM}_{2.5}$ composition in the $\mathrm{SE}$ US may shed further light on the sources and evolution pathways for the much-speculated HULIS species.

[5] This work addresses these questions by analyzing the organic fraction, especially the polar components, in fine aerosols in the SE US. A suite of analytical instruments are used concurrently for this purpose. Back trajectories of

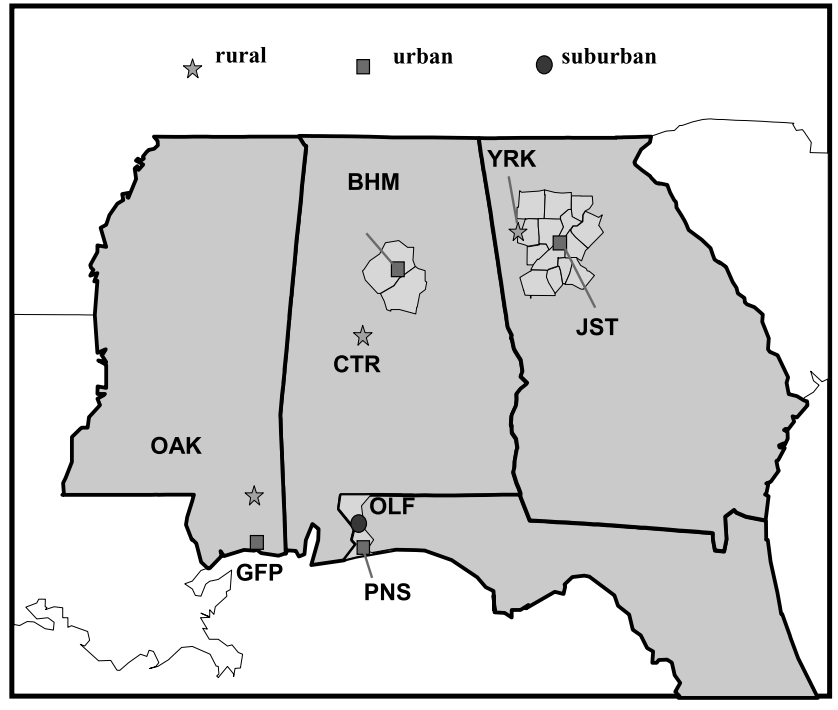

Figure 1. SEARCH network. Atlanta (JST), north Birmingham (BHM), Centreville (CTR), and Pensacola (PNS) are the four sampling sites used for analysis in this work.

specific samples and correlations among chemical and field variables are also examined. Possible sources and evolution pathways of the identified organic species are then discussed.

\section{Experiment}

\subsection{Filter Sampling and Field Measurements}

[6] The quartz and Teflon filters used for this work were collected at four different sites in the SEARCH network on 4 days in June 2004. Twenty-four-hour composite samples were taken; owing to this long sampling time, the contribution of adsorbed compounds to the total organic aerosol mass can be assumed to be relatively low [Turpin et al., 1994]. Detailed descriptions of the four sites, including their terrain, vegetation, industrial and transportation sources and surrounding environment, are described in a SEARCH overview paper [Hansen et al., 2003]. Briefly, JST (Jefferson Street, near downtown Atlanta, Georgia) and BHM (North Birmingham, Alabama) are both urban sites with mainly industrial and residential settings. CTR (Centreville, Alabama) is a rural site with a heavily wooded forest setting approximately $80 \mathrm{~km}$ southwest of BHM. PNS (Pensacola, Florida) is an urban site with a mainly residential setting. Figure 1 shows the location of these four sites, along with other SEARCH sites. It is worth noting that JST, BHM and CTR are all inland sites, whereas PNS is a coastal site subject to frequent prevailing winds from the Gulf of Mexico.

[7] Detailed descriptions of the measurements of gasphase species, particulate sulfate, nitrate, ammonium, major metal oxides, total organic matter (TOM) and black carbon (BC) are also provided by Hansen et al. [2003], and will not be reiterated here. In particular, the TOM mass is estimated from the total organic carbon mass (section 3.5), which, in turn, is derived from thermal evolution measurements of 
total carbon and aethalometer measurements of BC with further corrections from the backup filter data.

\subsection{Filter Extraction and Sample Preparation}

[8] Quartz and Teflon filters collected from each of the four SEARCH sites were extracted in HPLC-grade methanol by $40 \mathrm{~min}$ of sonication. The filters were then removed from the methanol sample extract and stored at $-20^{\circ} \mathrm{C}$ with dichloromethane added for archiving purposes. In order to remove soot and filter media residues, the filter extracts were centrifuged for $10 \mathrm{~min}$ at $4500 \mathrm{rpm}$ and then filtered through a PALL Life Sciences Acrodisc CR 25-mm syringe filter (PTFE membrane, 0.2- $\mu \mathrm{m}$ pore size) into a scintillation vial. Because these samples usually contain a high concentration of inorganic ions such as sulfate, a solid phase extraction (SPE) technique was applied to desalt and further pretreat the sample extracts. This procedure used 3-mL Supleco Discovery $\mathrm{C}_{18}$ reverse phase SPE tubes. Following the sonication, centrifuging and filtering steps outlined above, filter extracts were blown to near dryness with a gentle stream of $\mathrm{N}_{2}$ and were reconstituted with $5 \mathrm{~mL}$ of $1 \%$ acetic acid in water. The $1 \%$ acetic acid buffer was used to adjust the $\mathrm{pH}$ of the extracts to allow many organic species to become protonated and thus better retained on the $\mathrm{C}_{18}$ material upon sample addition to the SPE tube. Three milliliters of water was then washed through the SPE tube to desalt the sample. Organic species were then gradually eluted off the SPE tube with $5 \mathrm{~mL}$ of methanol. This eluate was collected, blown dry, and reconstituted with $0.1 \%$ acetic acid in $50 \%$ water $/ 50 \%$ methanol. Field blank filters and lab control filters were extracted and pretreated the same way as the samples. Aliquots of each filter extract were subsequently analyzed by the following four analytical techniques.

\subsection{LC-MS Analysis}

[9] A Hewlett-Packard 1100 Series HPLC-single quadrupole MS system, equipped with an electrospray ionization (ESI) source, was used to identify and quantify relatively polar, acidic species in SEARCH 2004 aerosol samples, under the negative ion mode. A Waters Nova-Pak $\mathrm{C}_{18}$ column $(3.9 \times 300 \mathrm{~mm})$ was used to separate the organic species before the MS detection. The eluents used were $0.1 \%$ acetic acid in water (A) and methanol (B). A 40-min gradient elution program was used, where eluent $\mathrm{B}$ increased from $10 \%$ to $90 \%$ in the first $35 \mathrm{~min}$ and then decreased to $10 \%$ in 5 min. During full spectrum MS mode of analysis, the fragmentor voltage was set at $60 \mathrm{~V}$ and most species were detected as their molecular ions. During the upfront CID (i.e., collision-induced dissociation) mode of analysis, the fragmentor was set at $110 \mathrm{~V}$ resulting in partial fragmentation of the molecular ions. By comparing the two sets of MS data and their different fragmentation patterns, some structural information on the analyzed species was gleaned.

\subsection{Ion Trap MS (ITMS) Analysis}

[10] Another aliquot of the filter extract was analyzed by a Finnigan LCQ ion trap mass spectrometer (equipped with an ESI source) without chromatographic separation. Under the negative ion mode, relatively polar species with MW up to 1200 were detected. Under the positive ion mode, a broader range of compounds with MW up to 1600 Da were detected as mainly $\mathrm{Na}^{+}$adducts. By comparing the detection under both ion modes, aerosol components of different polarity and acidity can be assessed. In addition, specific ions of interest were isolated from the rest of the sample matrix and were further fragmented to produce tandem mass spectra, aiding structure elucidation.

\subsection{LDI MS Analysis}

[11] A third aliquot of the filter extract was analyzed by laser desorption/ionization mass spectrometry (LDI MS) on porous silicon (i.e., DIOS). In DIOS, a porous Si surface serves as LDI matrix while in standard matrix-assisted LDI (MALDI), a UV-light absorbing species is the matrix. DIOS-LDI MS was used in favor of MALDI in order to avoid interferences from MALDI matrix ions. After the extract was dried on the DIOS chip, the sample plate was analyzed by a Voyager-DE PRO MALDI time-of-flight (TOF) mass spectrometer in order to assess the MW range of aerosol components. Analyses were performed in the linear mode with delayed extraction on. The laser intensity was adjusted at $\sim 10 \%$ above ionization threshold to permit detection without inducing fragmentation. Usually, 900 laser shots were summed to get a representative spectrum of a sample.

\subsection{High-Resolution MS Analysis}

[12] A fourth aliquot of the filter extract was analyzed by a Waters LCT Premier electrospray time-of-flight mass spectrometer in the Department of Chemistry at the University of California, Irvine, operated in the negative ion mode using $\mathrm{W}$ geometry. Initial calibration used sodium formate clusters with a known compound spiked into the analytical sample for lock-mass corrections to obtain accurate mass for the ions with $\mathrm{m} / \mathrm{z} 294$.

[13] For further confirmation, a JEOL JMS-600H doublefocusing, high-resolution, magnetic sector mass spectrometer at Caltech was also used for accurate mass measurements of the ion with $m / z$ 294. The sample was analyzed by Fast Atom Bombardment (FAB) in the negative ion mode with instrument resolving power set to 3000 , using the $10 \%$ valley definition. For these experiments the instrument was first externally calibrated with polyethylene glycol (PEG) clusters in the negative ion mode. The sample was mixed with glycerol, and glycerol cluster ions were used for internal calibration of the mass spectrum.

\section{Results and Discussion}

\subsection{Identification of Organic Components in Fine} Aerosols

[14] All four analytical methods described in section 2 were used to identify organic species in the fine aerosols in the SE US. As we demonstrate next, these measurements indicate possible structures of specific species and reveal a consistent picture of the organic aerosol composition.

[15] Figure 2 shows the total ion chromatogram (TIC) and several $(\mathrm{m} / \mathrm{z} 171,185,294,313)$ extracted ion chromatograms (EIC) of a typical $\mathrm{PM}_{2.5}$ sample collected during SEARCH 2004. Background signals from field blanks are subtracted from all aerosol samples for identification and quantification. It is noticeable that multiple peaks are present in the EIC of certain ions, such as those with $\mathrm{m} / \mathrm{z}$ 

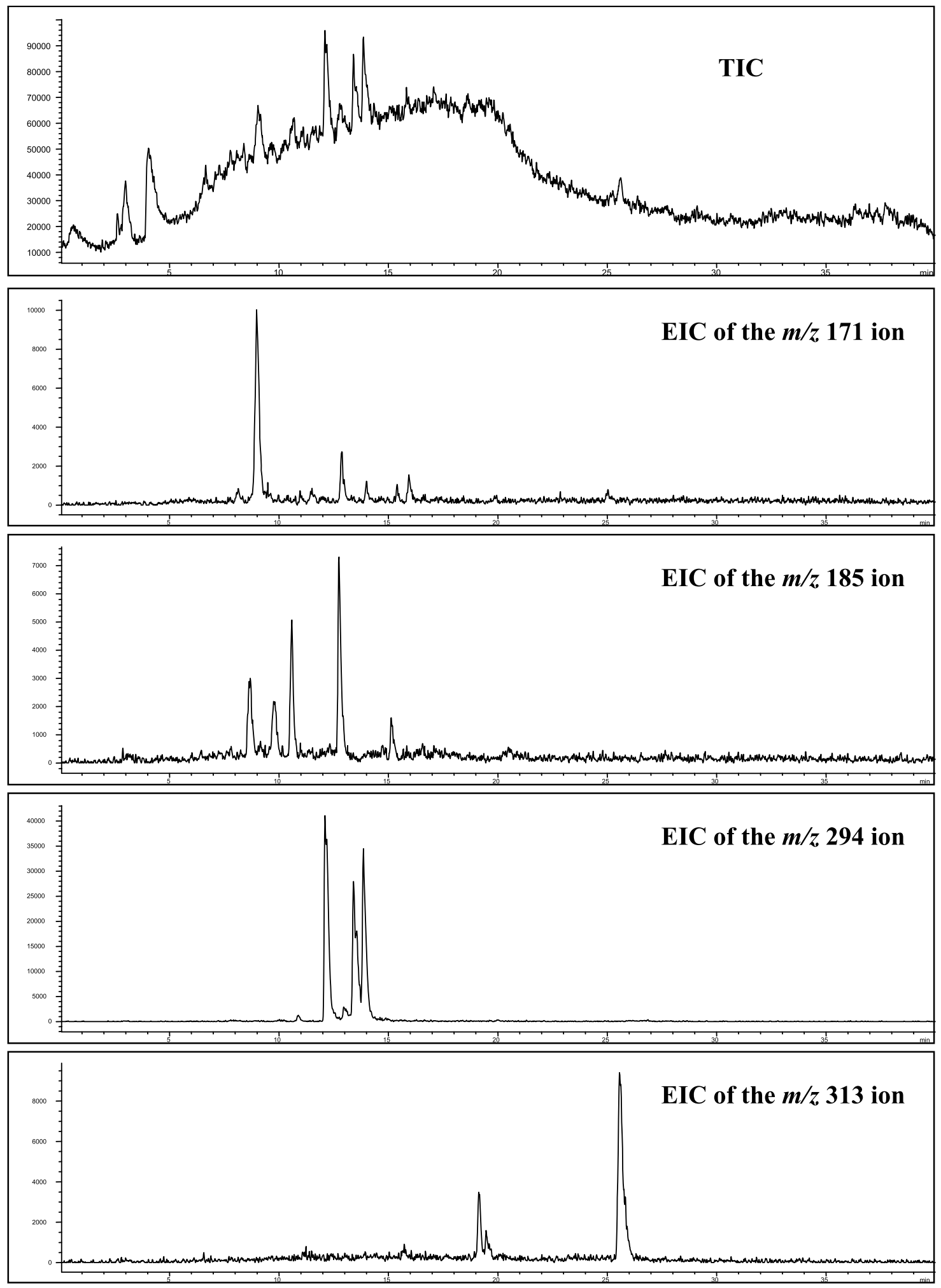

Figure 2. TIC and several EIC of the extract of the $\mathrm{PM}_{2.5}$ sample collected at the BHM site on 23 June 2004. The $y$ axis is intensity for the TIC and all EICs.

171, 185, 187, 294, 313, and 342. These same-nominal mass species could have either different elemental compositions or different isomeric structures with the same elemental composition, which is not possible to pinpoint owing to the unit mass resolution in the quadrupole MS. Throughout this paper, each set of these same-mass species detected by the LC-MS shall be called "isobaric species" or "isobaric compounds," terms commonly used in the mass 


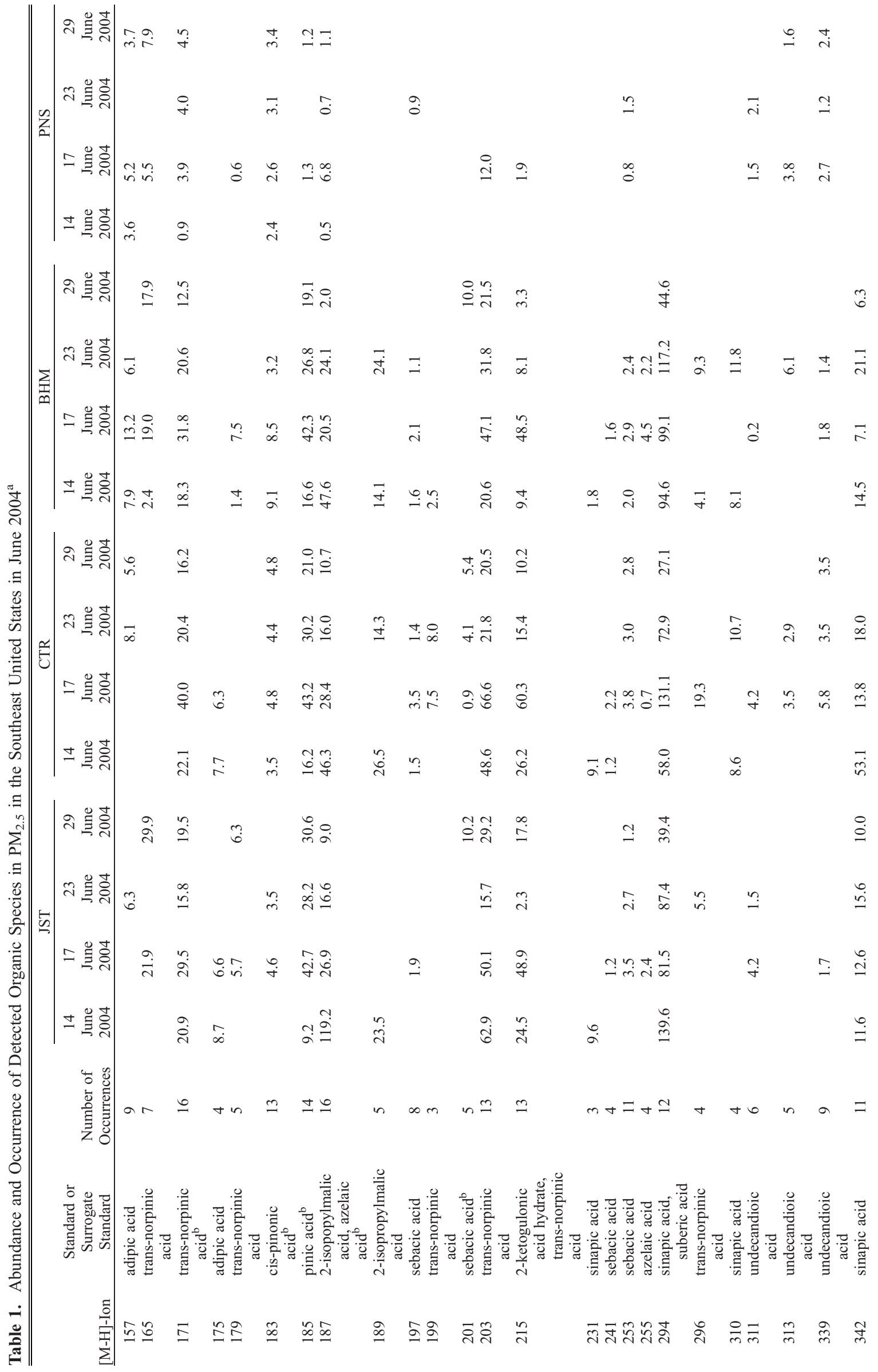




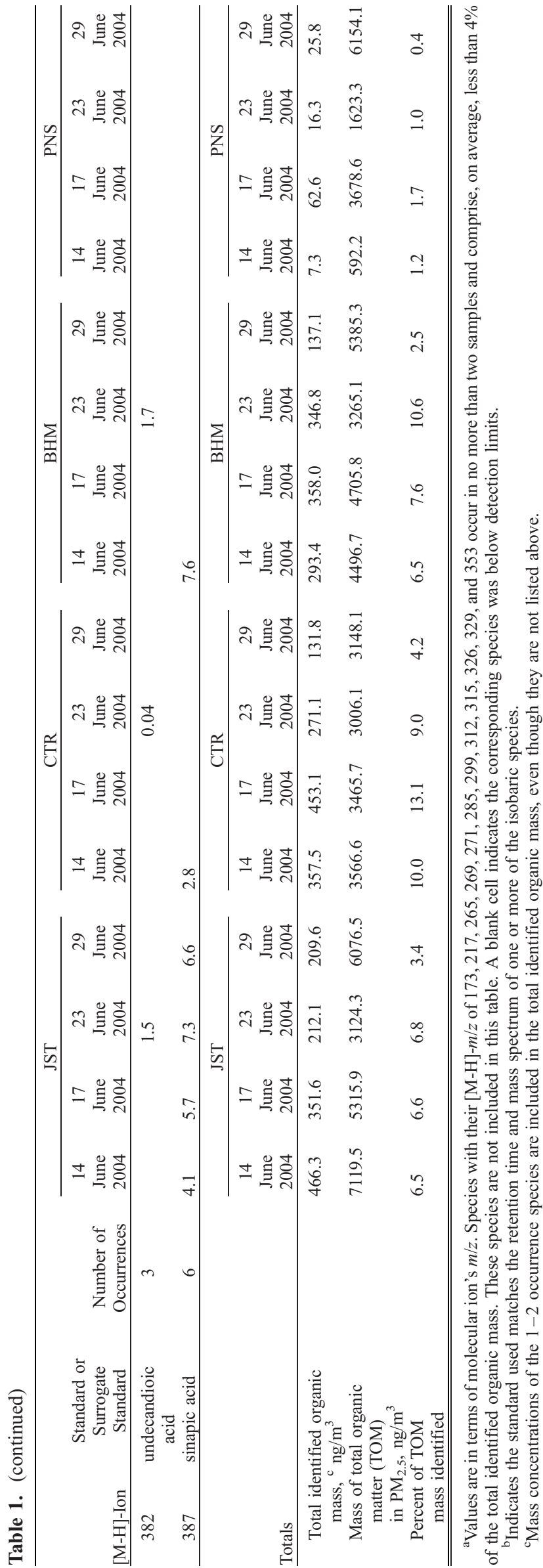

spectrometry community. We note that this chemical definition of isobaric (of equal molecular weight) is different from and should not be confused with the meteorological definition of isobaric (of equal atmospheric pressure). The presence of these isobaric species indicates the complexity of the organic fraction of $\mathrm{PM}_{2.5}$ in the SE US, and they can originate from either isomerization through a single reaction pathway or multiple sources and reaction pathways. We will discuss these isobaric species further in section 3.2.

[16] A series of standard compounds were analyzed by the LC-MS to obtain their retention times and ESI (negative ion mode) mass spectra. The reproducibility of these retention times is mostly within $0.05 \mathrm{~min}$ and never exceeds $0.1 \mathrm{~min}$. The details of standard calibration on the same LCMS and the relationship between retention time and compound structure are discussed in detail by Gao et al. [2004a]. Owing to the "soft" nature of the ESI source, all standard compounds are detected as deprotonated molecular ions $[\mathrm{M}-\mathrm{H}]^{-}$and display no or minimal fragmentation in the mass spectra. Earlier work has shown that acidic compounds with MW below 600 tend to form only singly charged ions (mainly $[\mathrm{M}-\mathrm{H}]^{-}$) in the ESI-MS [Leenheer et al., 2001; Kiss et al., 2003]. It is then reasonable to assume that the detected organic species in the fine aerosols in SEARCH 2004 are mostly in the form of $[\mathrm{M}-\mathrm{H}]^{-}$. Each chromatographic peak in the TIC in Figure 2 represents either a single species or several convoluted species due to co-eluting. From the EIC, 40 distinct species are detected in the 16 SEARCH 2004 $\mathrm{PM}_{2.5}$ samples, which are listed in Table 1 except for those species that are detected in only one or two samples (i.e., occurrence $<3$ ). Some individual isobaric species are not listed in this table for two reasons. The analytical techniques in this work cannot pinpoint the exact structures of most of these isobaric species. In addition, as will be shown in section 3.2, in the sampling region in June 2004, there is a rather constant mass distribution pattern of each set of isobaric species, rendering a summation within each set still a good representation of a specific species.

[17] It is of great interest to understand the molecular structures of these species, which can shed light on their sources and formation pathways. For a species that matches the retention time and mass spectrum of a certain standard compound, the structure of the standard is assigned to the corresponding species. For example, certain isobaric species of $\mathrm{m} / \mathrm{z} 171,183$, and 185 (all detected as $[\mathrm{M}-\mathrm{H}]^{-}$) correspond to the standards of norpinic acid, pinonic acid, and pinic acid, respectively. The two-stage tandem mass spectra by the ITMS (MS/MS) of these species are similar to those of the standards, further confirming their structures. Previous work has shown that these organic acids are some of the dominant low-MW products from gas-phase monoterpene oxidation, such as $\alpha$-pinene ozonolysis [Yu et al., 1999; Glasius et al., 2000; Gao et al., 2004b]. Considering the substantial presence of terpene-emitting vegetation, such as mixed conifer and deciduous forests, in the SE US, terpene oxidation is a very likely pathway to form these species in $\mathrm{PM}_{2.5}$ there.

[18] There are other species detected by the LC-MS for which standards are not available for comparison, making elucidation of their structures more difficult. However, as will be discussed in section 3.4, there is good correlation between many other species (e.g., $\mathrm{m} / z$ 203, 215, 253, 294, 


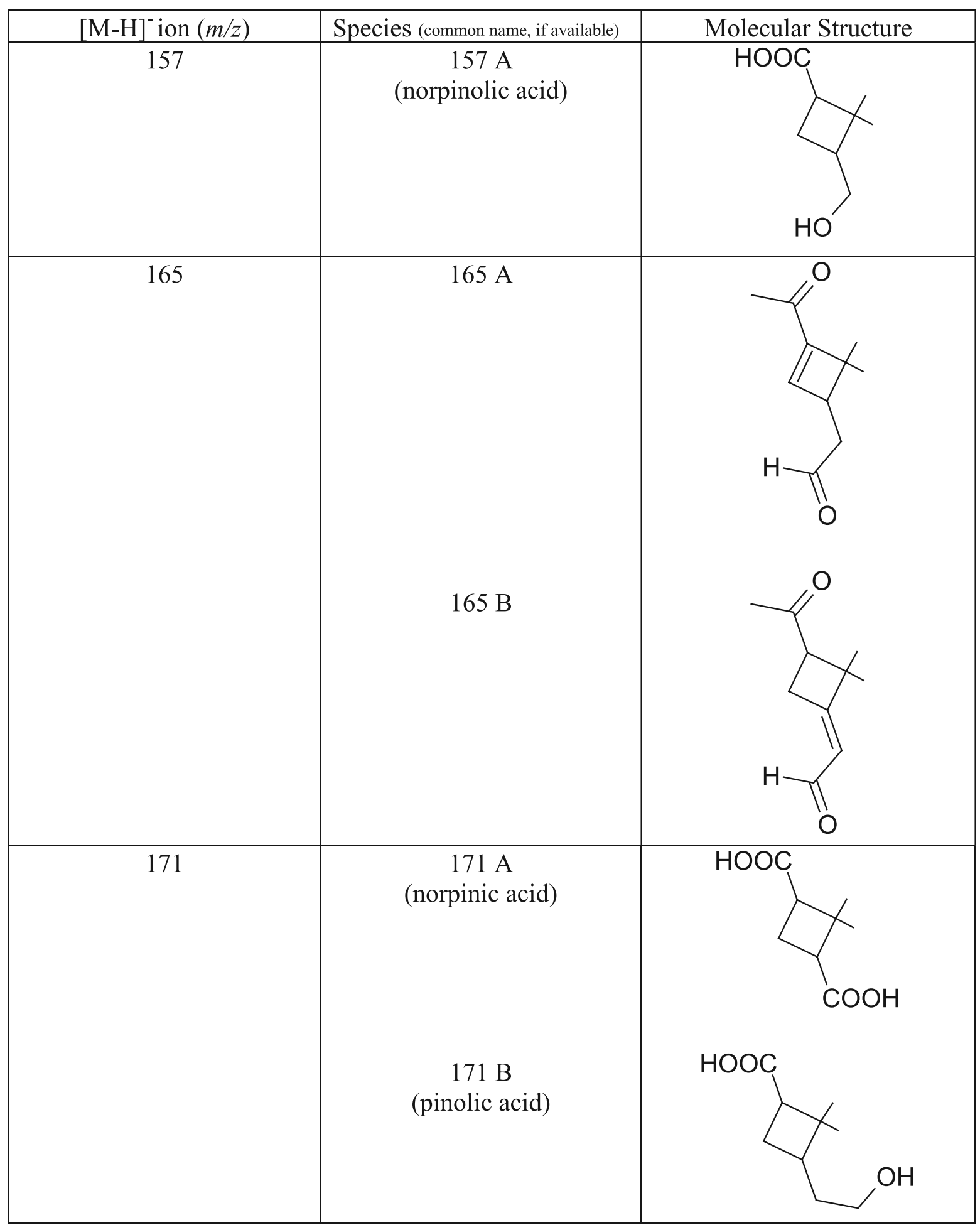

Figure 3. Identified and proposed molecular structures of some detected species $(\mathrm{MW}<250 \mathrm{Da})$ in $\mathrm{PM}_{2.5}$ in the SE United States in June 2004 (all drawn in the form of neutral species).

and 339 ions) and those likely with a biogenic source (e.g., $\mathrm{m} / \mathrm{z} 171,183$, and 185 ions). This suggests that the former may also come from terpene oxidation, at least for selected isobaric species. Most of them appear to contain carboxyl groups, as revealed by the MS/MS fragmentation patterns. Their rather early retention times on the $\mathrm{C}_{18}$ reversed-phase column, compared with those of known standards, suggest they are highly oxidized species. For species with MW below $250 \mathrm{Da}$, we propose possible structures based on their MS/MS fragmentation patterns and mechanistic consideration of monoterpene oxidation (Figure 3). Some of these structures have been proposed before, such as by 


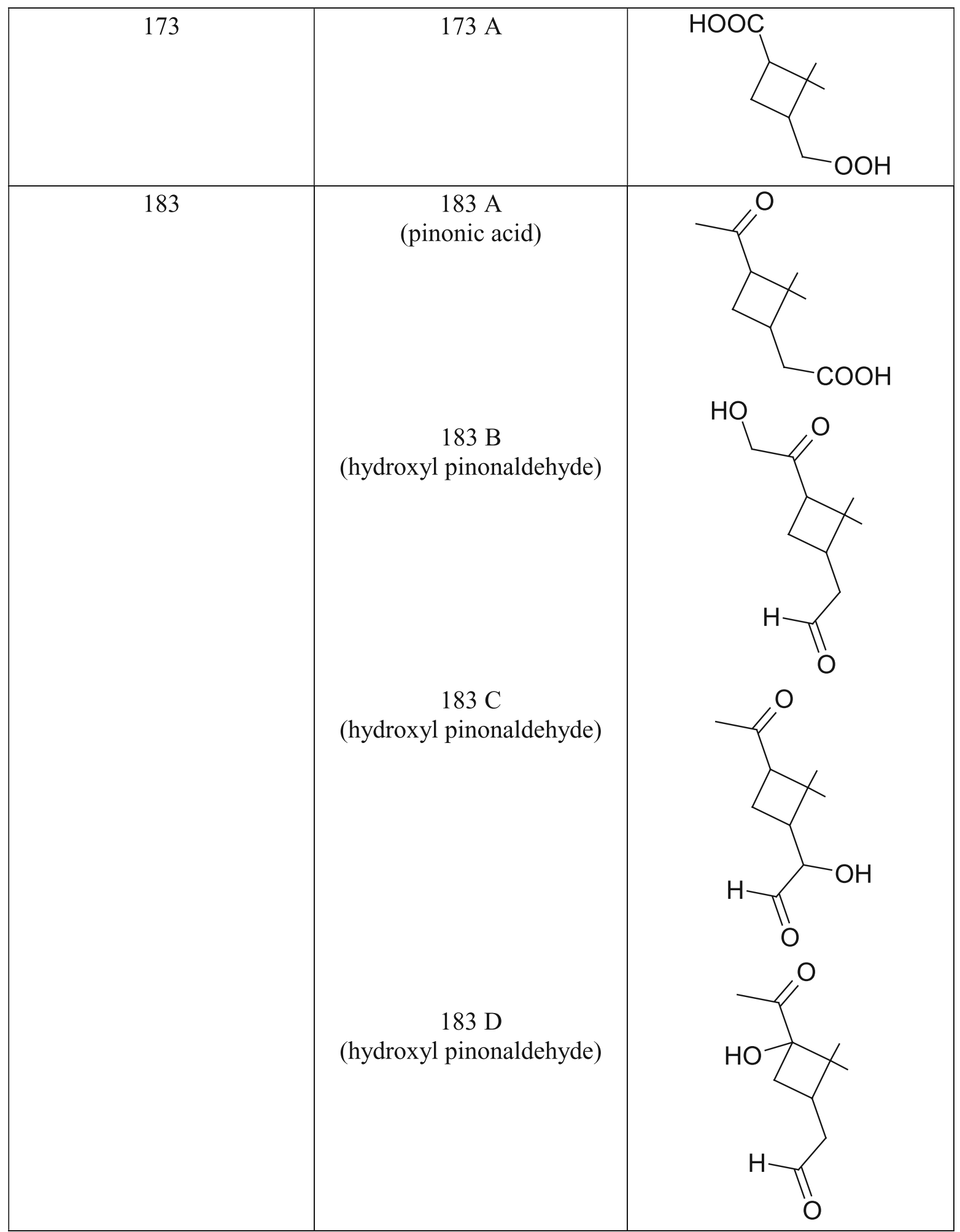

Figure 3. (continued)

Winterhalter et al. [2003], but they require further confirmation such as by the high-resolution MS. For species with MW above $250 \mathrm{Da}$, it is generally difficult to even propose possible structures. However, since the $\mathrm{m} / \mathrm{z} 294$ ion is almost always the most abundant species (except for the
PNS site), comprising $19-41 \%$ ( $28 \%$ on average) of the total identified organic mass, we attempt to elucidate its structure by several other approaches.

[19] The fact that the $\mathrm{m} / \mathrm{z} 294$ molecular ion is evennumbered suggests, at first glance, that it may be a 


\begin{tabular}{|c|c|c|}
\hline 185 & $\begin{array}{c}185 \mathrm{~A} \\
\text { (pinic acid) } \\
\text { (pinalic peroxo acid) } \\
185 \mathrm{~B} \\
185 \mathrm{C}\end{array}$ & (O)OH \\
\hline 187 & $\begin{array}{c}187 \mathrm{~A} \\
\text { (azelaic acid) } \\
187 \mathrm{~B} \\
\text { (norpinic peroxy acid) }\end{array}$ & $\mathrm{HOOC}-\left(\mathrm{CH}_{2}\right)_{7}-\mathrm{COOH}$ \\
\hline 197 & $\begin{array}{c}197 \mathrm{~A} \\
\text { (keto-pinonic acid) } \\
197 \mathrm{~B} \\
\text { (keto-pinonic acid) }\end{array}$ & HOOC \\
\hline
\end{tabular}

Figure 3. (continued) 


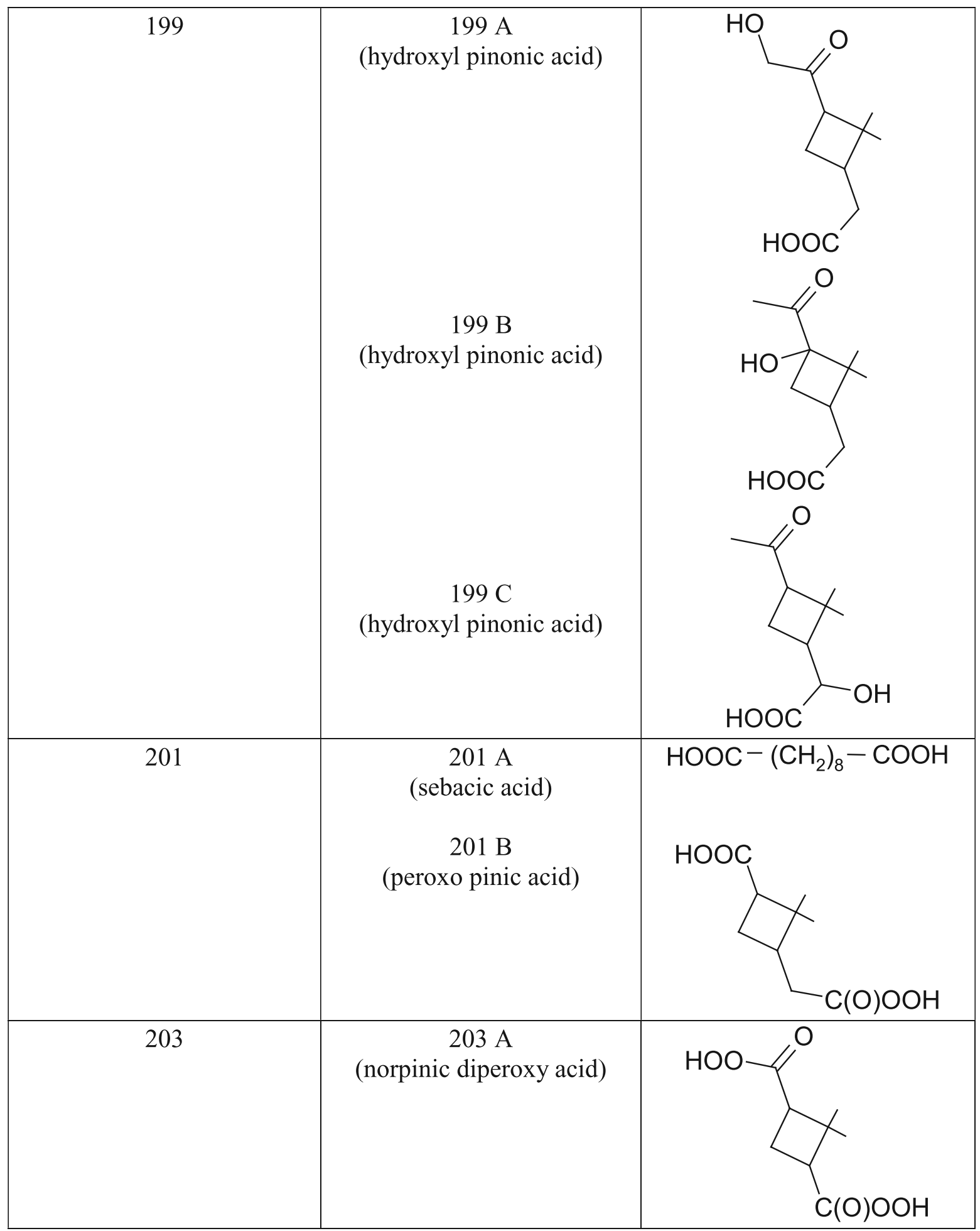

Figure 3. (continued)

nitrogen-containing species. This hypothesis is supported by the MS/MS data. Figure 4 shows the ion trap MS/MS of the $\mathrm{m} / \mathrm{z} 294$ ion from the same samples as shown in Figure 2, where all isobaric species were isolated and fragmented simultaneously. The most abundant fragment ion $(m / z=231)$ corresponds to a loss of 63 , which is likely $\mathrm{HNO}_{3}$. Another fragment ion $(m / z=247)$ corresponds to a loss of 47 , which is likely $\mathrm{HNO}_{2}$. As will be discussed in section 3.4, there is good correlation between the abundance of the $\mathrm{m} / \mathrm{z} 294$ ion and that of the $\mathrm{m} / \mathrm{z}$ $171,185,187,197,203$ and 215 ions, the latter likely formed via terpene oxidation. It follows that $\mathrm{m} / \mathrm{z} 294$ ion 


\begin{tabular}{|c|c|c|}
\hline 215 & $\begin{array}{l}215 \mathrm{~A} \\
\text { (undecanedioic acid) } \\
\text { 215 B } \\
\text { (hydroxy pinonic peroxy } \\
\text { acid) } \\
\\
\text { (hydroxy pinonic peroxy } \\
\text { acid) } \\
\\
\text { (hydroxy pinonic peroxy } \\
\text { acid) }\end{array}$ & (OH \\
\hline 217 & $\begin{array}{c}217 \text { A } \\
\text { (hydroxy pinic peroxy acid) } \\
217 \text { B } \\
\text { (hydroxy pinic peroxy acid) }\end{array}$ & $\mathrm{C}(\mathrm{O}) \mathrm{OOH}$ \\
\hline
\end{tabular}

Figure 3. (continued)

may also come from terpene oxidation, but with the participation of $\mathrm{NO}_{\mathrm{x}}$ to account for the presence of $\mathrm{HNO}_{2}$ and $\mathrm{HNO}_{3}$ in its structures. It is worth noting that Kiss et al. [2003] also observed an $\mathrm{m} / \mathrm{z} 294$ ion in their
LC-MS analysis of rural fine aerosols, which they concluded as different from the HULIS fraction that exhibited a polyconjugated nature similar to aquatic fulvic acids [Kiss et al., 2001]. The secondary formation scheme 


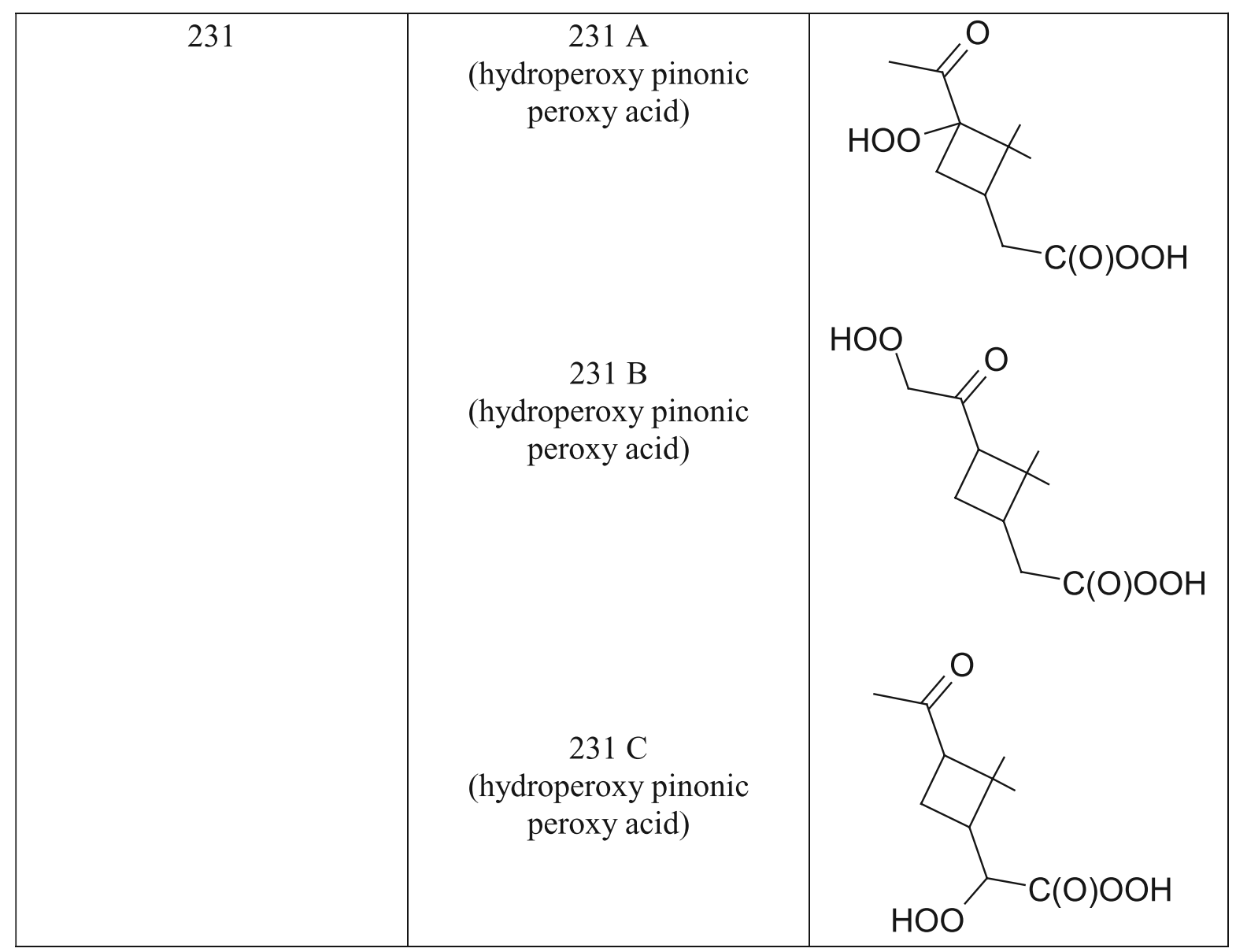

Figure 3. (continued)

proposed here appears to be a viable pathway. Since the organic eluent used by Kiss et al. [2003] was isopropanol, whereas it is methanol in this work, this is confirmation that the $\mathrm{m} / \mathrm{z} 294$ ion is not an eluent adduct.

[20] The upfront CID MS/MS analysis of the $\mathrm{m} / \mathrm{z} 294$ ion by the LC-MS provides a consistent picture. Figure 5 shows the EIC of the $m / z 294$ ion and the upfront CID mass spectra of the three main chromatographic peaks, with the fragmentor voltage set at $110 \mathrm{~V}$. It can be seen that the $\mathrm{m} / \mathrm{z} 247$ ion is a major fragment ion in peak 1 and peak 2, while the $\mathrm{m} / \mathrm{z} 231$ ion is a major fragment ion in peak 3. This suggests HONO is a dominant loss from the $\mathrm{m} / \mathrm{z} 294$ ion eluting in the first two peaks, while $\mathrm{HONO}_{2}$ is a dominant loss from the species eluting in the third peak. In the full spectrum mode of the LC-MS analysis (fragmentor voltage $=60 \mathrm{~V}$ ) as shown in Figure 6, the $\mathrm{m} / \mathrm{z} 294$ ion elutes similarly in three main peaks, but with much higher intensities due to much less fragmentation. Strikingly, in addition to the dominant molecular ion, isotopic ions with $\mathrm{m} / \mathrm{z}$ of 295 and 296 are always present in the mass spectra. The distinct pattern of isotopic distribution strongly suggests the $\mathrm{m} / \mathrm{z}$ 294 ion is also a sulfur-containing species. Also strikingly, the $\mathrm{m} / \mathrm{z} 96$ ion is always the dominant ion in the upfront CID mass spectra of the $\mathrm{m} / \mathrm{z} 294$ ion, next to a smaller $\mathrm{m} / \mathrm{z} 97$ ion. These two fragment ions are probably attributed to $\mathrm{SO}_{4}^{-}$and its isotope (or $\mathrm{HSO}_{4}^{-}$), which are also seen in the ion trap MS/MS spectrum (Figure 4). However, neither is present in the full spectrum mode LC-MS spectra. This strongly suggests that there is an $\mathrm{SO}_{4}$ structure in the $\mathrm{m} / \mathrm{z} 294$ species, and it is likely covalently bonded with the rest of the $\mathrm{m} / z 294$ ion. Upon CID fragmentation, $\mathrm{SO}_{4}^{-}$comes off as a radical ion, which has been suggested to react with monoterpenes to form $\mathrm{SO}_{4}^{-}$adducts in oxygen-poor conditions [Buxton et al., 2000]. Other ions such as those with $\mathrm{m} / \mathrm{z} 171,185$, 203 and 215 only have a small $\mathrm{m} / \mathrm{z} 97$ daughter ion, which probably comes from externally mixed $\mathrm{HSO}_{4}^{-}$in the $\mathrm{PM}_{2.5}$ that entered the ion trap together with the organic parent ion.

[21] The high-resolution MS measurements on the LCT electrospray TOF instrument show the accurate mass of the $\mathrm{m} / \mathrm{z} 294$ ion in the sample in Figure 2 to be $294.0638 \mathrm{Da}$. This mass is consistent with the measurements on the magnetic sector instrument using FAB ionization. We emphasize that because there is no separation of the $\mathrm{m} / \mathrm{z} 294$ ions before the MS detection, this is the average mass of all possible $\mathrm{m} / \mathrm{z} 294$ ions present. Considering the likely presence of both $\mathrm{N}$ and $\mathrm{S}$, one possible molecular formula, with the smallest difference between the measured and calculated mass, is $\mathrm{C}_{10} \mathrm{H}_{16} \mathrm{NO}_{7} \mathrm{~S}$, which gives rise to the six possible structures listed in Figure 7. The attack of $\mathrm{OH}$ 


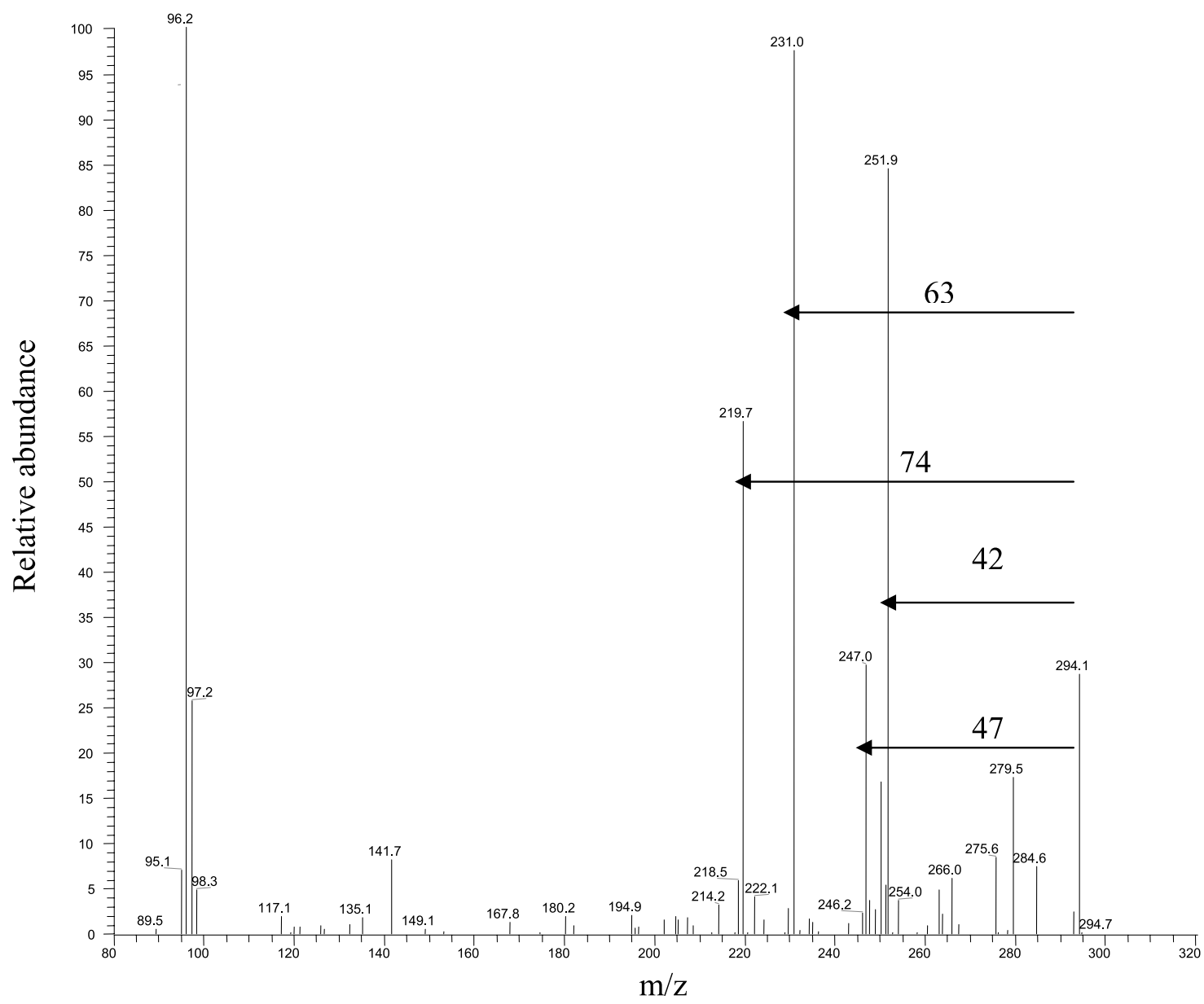

Figure 4. Ion trap MS/MS spectrum of the $m / z 294$ ion (containing all isobaric species) from the same sample as shown in Figure 2.

to $\mathrm{NO}$ and $\mathrm{NO}_{2}$ and the attack of $\mathrm{H}$ to $\mathrm{NO}_{2}$ and $\mathrm{NO}_{3}$ in these structures would lead to the loss of $\mathrm{HNO}_{2}$ and $\mathrm{HNO}_{3}$, respectively, explaining the $m / z 247$ and $m / z 231$ fragment ions in the ion trap MS/MS (Figure 4), upfront CID mass spectra (Figure 5), and the high-resolution MS/MS. Known monoterpene oxidation pathways can explain the formation of these species quite nicely. For example, the Criegee intermediate from $\alpha$-pinene ozonolysis can further react with $\mathrm{NO}_{\mathrm{x}}$ and $\mathrm{SO}_{2}$ at the end carbon and $\alpha$-carbon to form $\mathrm{N}$, S-containing species. Further oxidation of $\mathrm{S}$ is expected to eventually form the $\mathrm{SO}_{4}$ or $\mathrm{SO}_{3}$ groups in the proposed structures. Again, the high-resolution MS shows an isotopic distribution pattern of $\mathrm{m} / z$ 294, 295 and 296 ions that is characteristic of sulfur. However, until exact masses are obtained for each isobaric ion with $\mathrm{m} / \mathrm{z} 294$, we can only propose tentative structures such as those in Figure 7.

[22] Recent chamber experiments conducted jointly with the U.S. Environmental Protection Agency (E. Edney, T. Kleindienst) show that secondary organic aerosols readily form from the photooxidation of varying combinations of $\alpha$-pinene, toluene and isoprene. These SOA have been analyzed by the same LC-MS method as in this work. Strikingly, when $\mathrm{SO}_{2}$ and $\mathrm{NO}_{\mathrm{x}}$ are both present and the precursors include $\alpha$-pinene, the $m / z 294$ ion is always detected as an SOA component. The three main peaks of the $\mathrm{m} / \mathrm{z} 294$ isobaric ions elute at the same retention times as in the SEARCH samples. However, the $m / z 294$ ion is not present in the SOA in the absence of either $\mathrm{SO}_{2}$ or $\alpha$-pinene. Furthermore, the $m / z 247$ and $m / z 231$ ions are the major fragment ions in the upfront CID mass spectra of the three main 294 chromatographic peaks, again similar to the SEARCH samples. These results strongly support the hypothesis that the $m / z 294$ ions are formed via monoterpene oxidation with the participation of $\mathrm{NO}_{\mathrm{x}}$ and $\mathrm{SO}_{2}$. Of course, the exact nature of the reactions (the roles of ozonolysis and photooxidation as well as the distinction between gas-phase, particle-phase and heterogeneous reactions) remains to be investigated.

[23] Most of these species detected by the LC-MS are also detected by the ion trap MS in the negative ion mode. Figure 8 shows the ITMS of the extract of the same sample as shown in Figure 2. It can be seen that $\mathrm{m} / \mathrm{z} 171,185,187$, 203, 215, 253, 281, 294, 313 and 342 ions are the molecular ions of the dominant species, which are also detected by the LC-MS (Table 1). The relative abundances of these species also agree well between these two sets of measurements. Together, they indicate that the relatively polar organic species in the SE US in June 2004 cover a MW range of 150-400 Da. This is rather consistent with the earlier estimate of the average MW (i.e., 215-345) of polar organic components in rural fine aerosols in Hungary [Kiss et al., 2003]. 

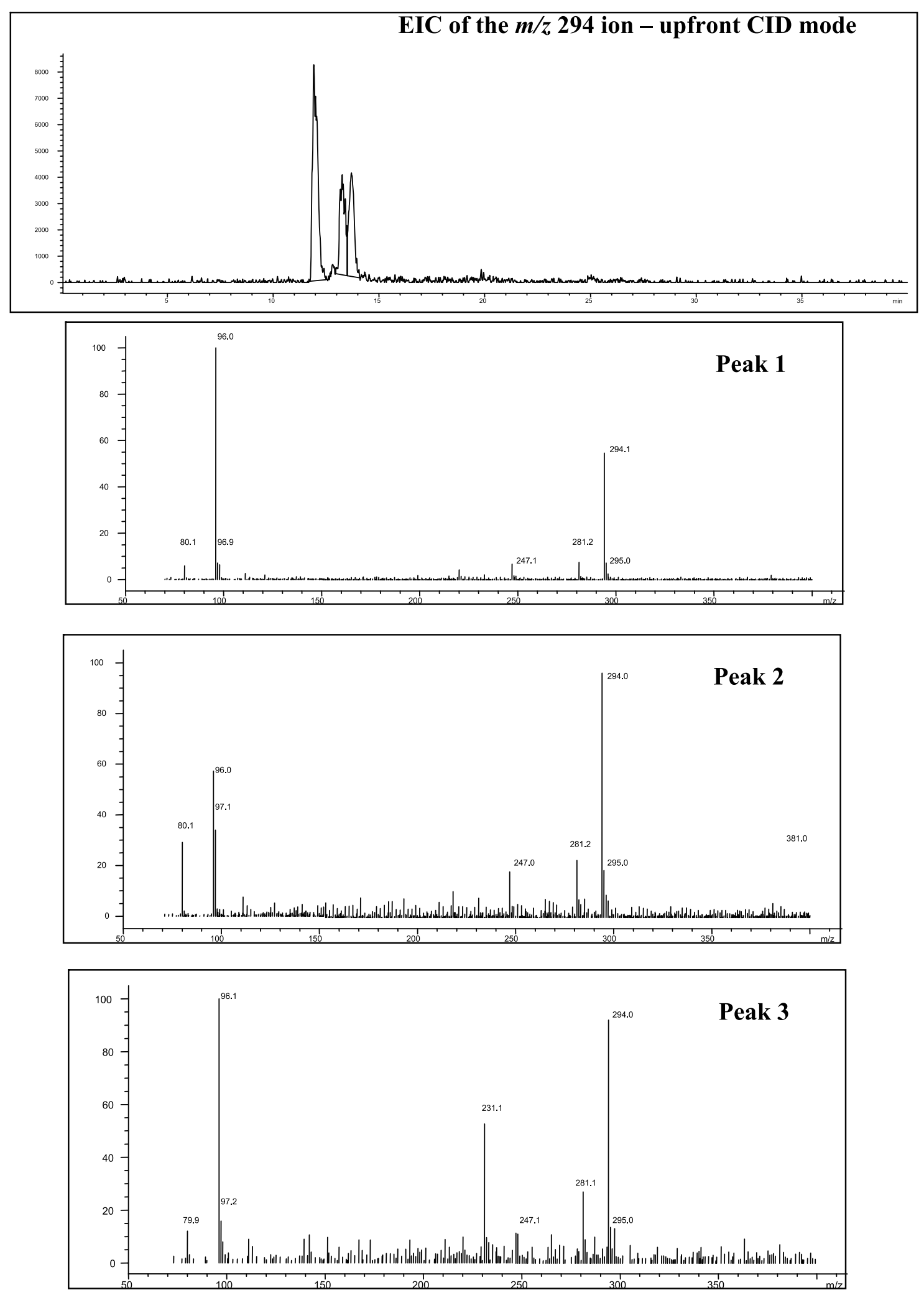

Figure 5. EIC of the $m / z 294$ ion in the same sample as shown in Figure 2 with the fragmentor set at $110 \mathrm{~V}$ (upfront CID mode), and the upfront CID mass spectra of the three main peaks (retention times = 12.0, 13.3 and 13.7 min for Peak 1, 2 and 3, respectively). The $y$ axis is intensity and relative abundance for the EIC and the mass spectra, respectively. 

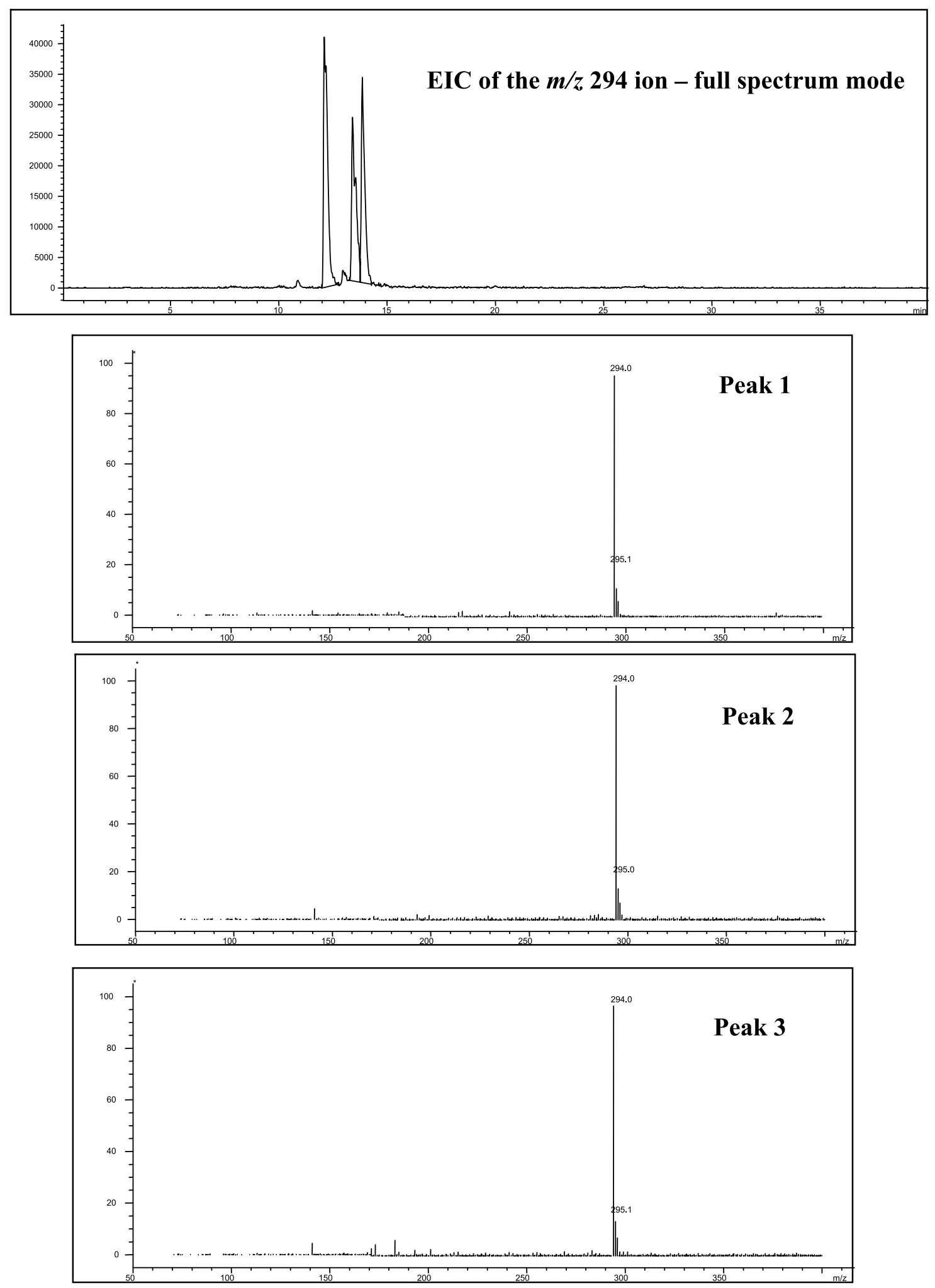

Figure 6. EIC of the $m / z 294$ ion in the same sample as shown in Figure 2 with the fragmentor set at $60 \mathrm{~V}$ (full spectrum MS mode), and the mass spectra of the three main peaks (retention times $=12.0,13.3$ and 13.7 min for Peak 1, 2 and 3, respectively). The $y$ axis is intensity and relative abundance for the EIC and the mass spectra, respectively. 


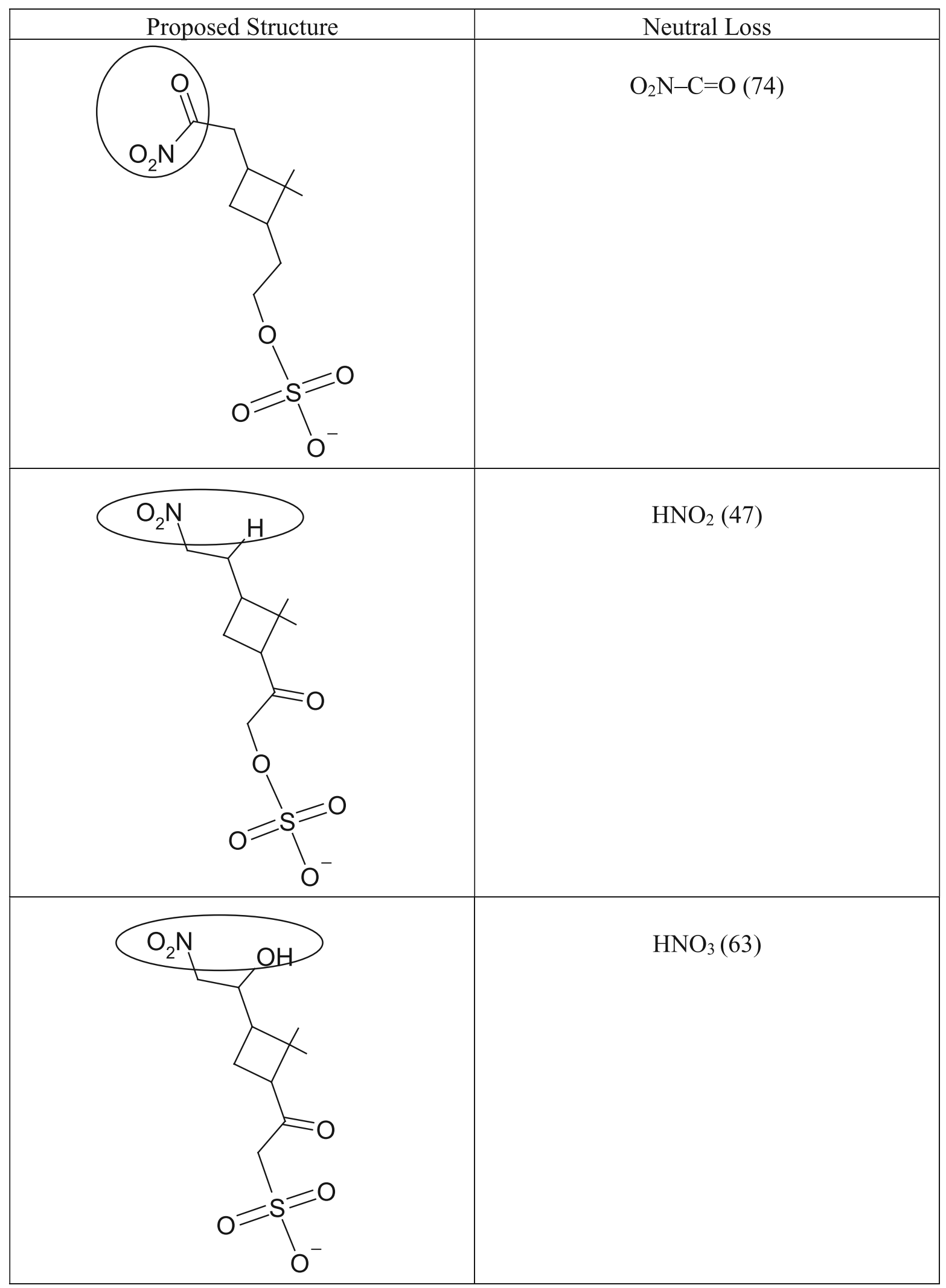

Figure 7. Proposed structures of the $m / z 294$ ion, based on the accurate mass measurements and the MS/MS fragmentation pattern in the ITMS and the upfront CID MS. 


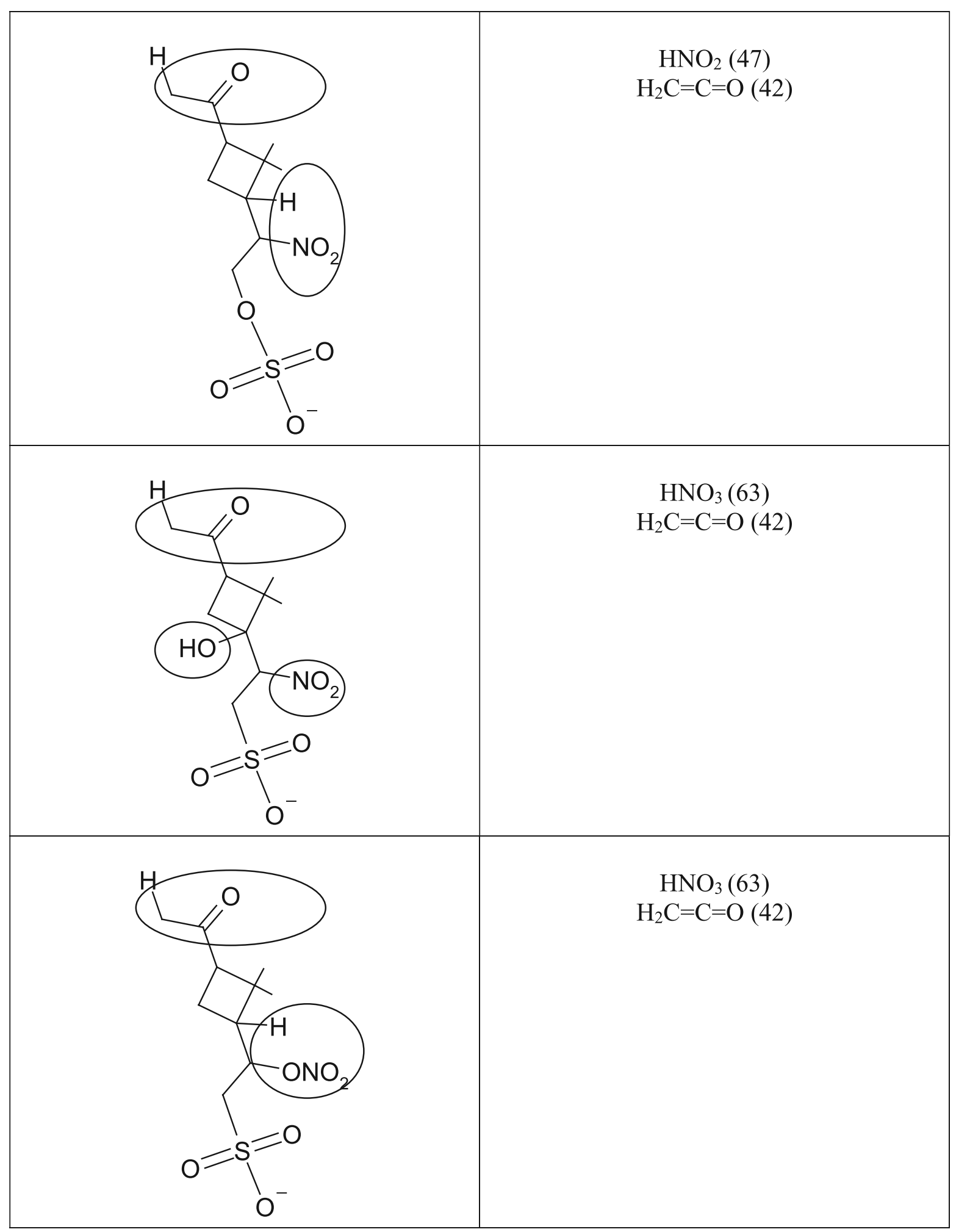

Figure 7. (continued) 


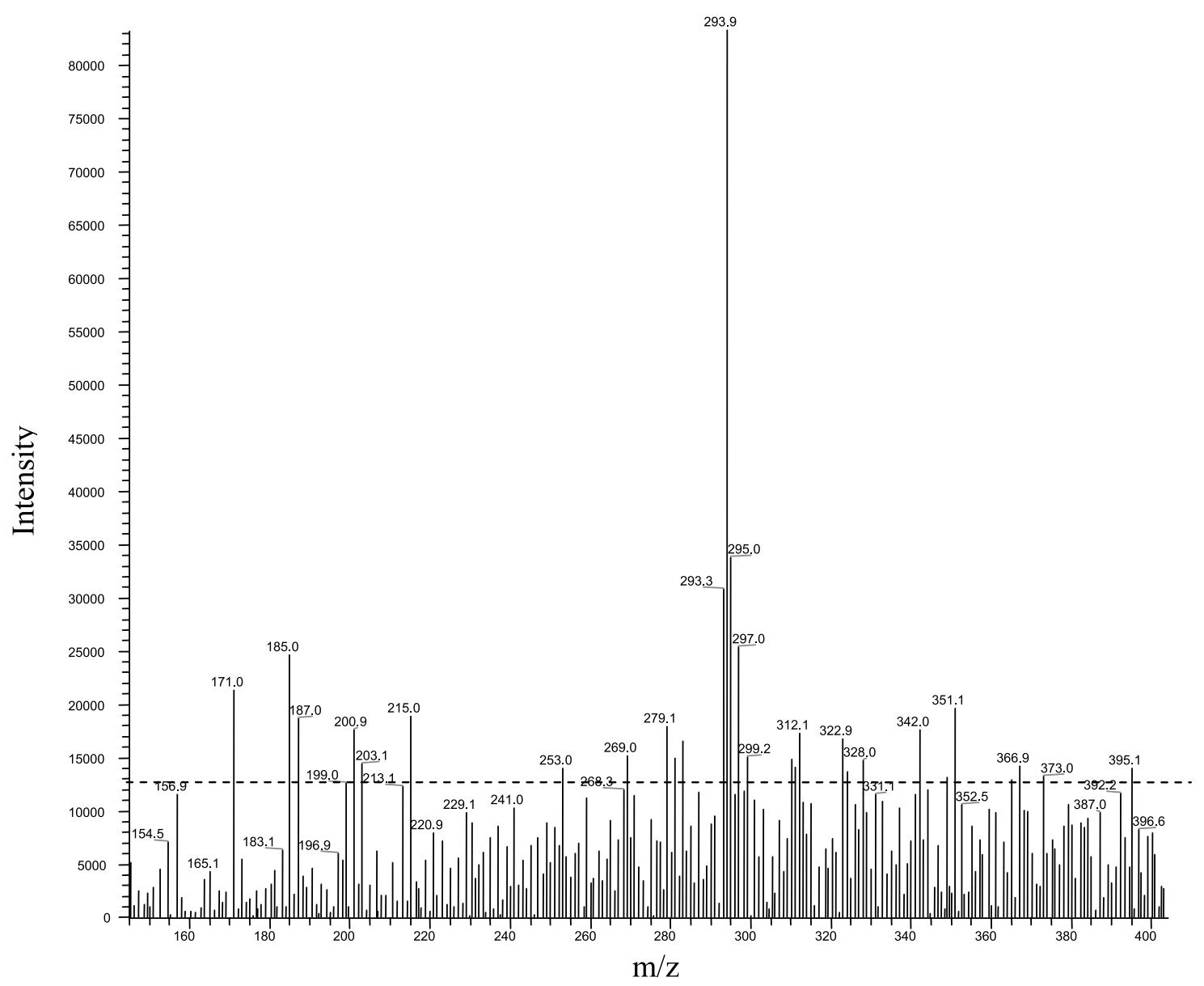

Figure 8. Ion trap mass spectrum (negative ion mode) of the same sample as shown in Figure 2. The detection limit was determined from field blanks and was $\sim 14,000$ as shown by the dashed line.

[24] In addition, LDI MS analyses of SEARCH 2004 samples also suggest that the majority of the detected organic species in $\mathrm{PM}_{2.5}$ cover the MW range of $150-$ $400 \mathrm{Da}$. For example, the LDI mass spectrum of the quartz filter sample collected at the JST site on 14 June 2004 is shown in auxiliary Figure $\mathrm{S} 1{ }^{1}$. A series of peaks separated by $14 \mathrm{Da}$ and $16 \mathrm{Da}$ are abundantly detected in the 150 to 350 mass range, and species with $\mathrm{m} / \mathrm{z}$ above 400 can be seen sporadically but at very low intensities. This is consistent with other ambient aerosol measurements by LDI MS, such as by Samburova et al. [2005]. Species with MW from 400 to $700 \mathrm{Da}$ detected in their study comprise only a small fraction of the total ion intensities, and these could be due to their distinct aerosol source (urban background).

[25] It is of particular interest to examine whether these identified species have an oligomeric nature, which is suggested recently for urban aerosols [Samburova et al., 2005]. As we have mentioned earlier, species with MW below $250 \mathrm{Da}$ are very likely oxidation products of monoterpenes, based on chemical and correlation analyses (section 3.4). Ion trap MS/MS analyses show that 18, 32, and 44 are the most common, dominant neutral losses from these species, which suggests they do not have a monomeric unit. Of species with MW from 250 to $400 \mathrm{Da}$, again, ion

${ }^{1}$ Auxiliary material is available at $\mathrm{ftp} / / \mathrm{ftp}$.agu.org/apend/jd/ $2005 \mathrm{jd} 006601$. trap MS/MS analyses show that the dominant neutral losses are usually small, such as 18, 44, 47, 63 and 74 . This does not support the presence of oligomers and corresponding monomeric fragments in the MS/MS spectra, such as those demonstrated by Gao et al. [2004a, 2004b]. A cautionary note here is that even though there is apparently regular mass difference (e.g., 14 and $16 \mathrm{Da}$ ) between identified species, such as the series of ions with $\mathrm{m} / \mathrm{z} 157,171,185$ / $187,201 / 203$, and 215 , or the series of ions with $\mathrm{m} / \mathrm{z} 294$, 310,326 and 342 , these species may simply be oxidation products that differ by $\mathrm{CH}_{2}$ (14), $\mathrm{O}$ (16) and $\mathrm{H}_{2} \mathrm{O}$ (18), arising from the complicated reaction pathways common in atmospheric oxidation. Species with MW above $250 \mathrm{Da}$ can, for example, come from oxidation of sesquiterpenes and even larger precursors. The ultimate criterion for the existence of oligomers should be whether repeating monomer (though not necessarily identical) units exist. Accordingly, in the LDI MS data (e.g., auxiliary Figure S1), the regularly spaced peaks with 14 and $16 \mathrm{Da}$ mass differences are mainly due to the various oxidation products differed by groups such as $\mathrm{CH}_{2}$ and $\mathrm{O}$. Therefore the polar organic species detected in $\mathrm{PM}_{2.5}$ in the SE US (MW 150-400 Da) do not appear to be oligomeric in nature. Similar species detected in rural aerosols and fogwater in Europe by other groups [e.g., Krivacsy et al., 2001; Kiss et al., 2003; Cappiello et al., 2003], often referred to as HULIS, are likely not oligomeric as well. However, for species with 
06/17/04 Samples

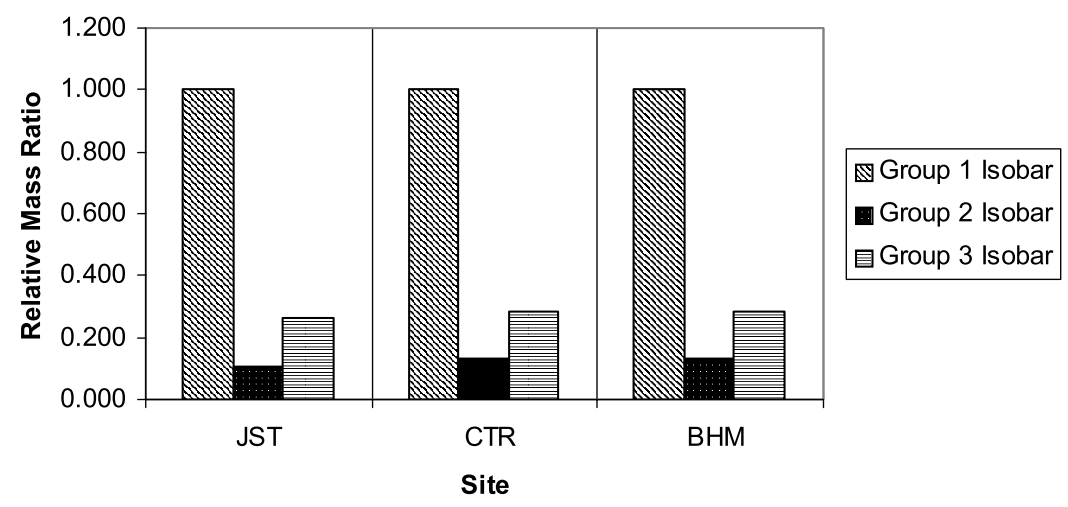

06/29/04 Samples

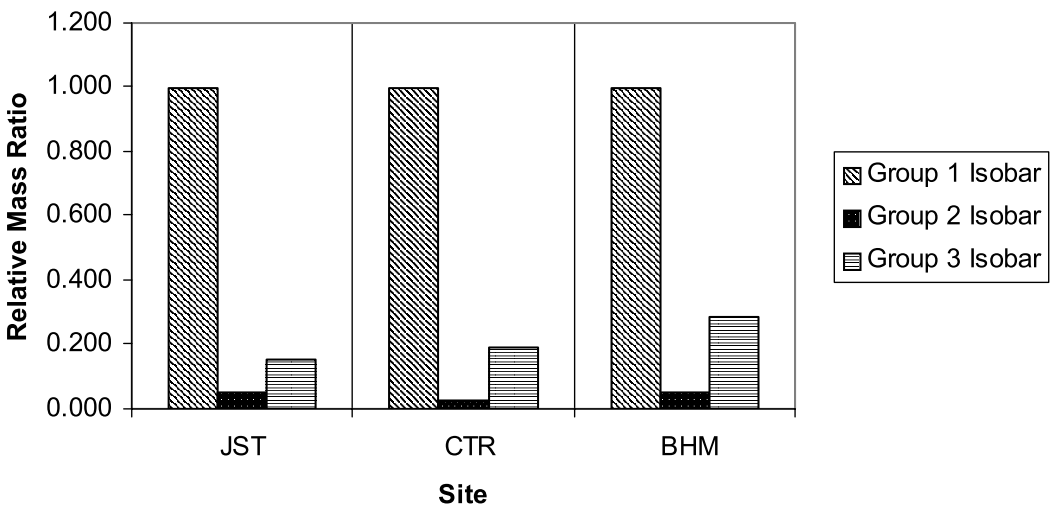

Figure 9. Relative abundance of three groups of 294 isobaric species, displaying a rather consistent distribution pattern.

MW above $400 \mathrm{Da}$, we cannot yet preclude the possibility that some are oligomers, originating from the first-generation oxidation products through the pathways suggested recently [Kalberer et al., 2004; Tolocka et al., 2004; Gao et al., 2004a, 2004b]. This will be discussed further in sections 3.4 and 3.5.

\subsection{Quantification of Organic Components in Fine Aerosols}

[26] Calibration factors were determined for each standard compound by carrying out a five-point calibration. These calibration factors are then used to quantify the detected species in the $\mathrm{PM}_{2.5}$ samples collected in the SEARCH network in June 2004. For those species matching the exact retention time (RT) and mass spectrum of a standard, that standard is used to quantify the mass concentration of the species. For example, cis-pinonic acid, pinic acid, and azelaic acid are used to quantify the $\mathrm{m} / \mathrm{z} 183$ ion $(\mathrm{RT}=14.0 \mathrm{~min})$, one of the $\mathrm{m} / \mathrm{z} 185$ isobaric ion $(\mathrm{RT}=$ $12.8 \mathrm{~min}$ ) and one of the $\mathrm{m} / \mathrm{z} 187$ isobaric ion $(\mathrm{RT}=15.8 \mathrm{~min}$ ), respectively. For those species without a match of standards, surrogate standards with similar retention times and molecular weights are used to quantify. More than $80 \%$ of the detected species have retention times within 1.0 min of their surrogate standards, while the rest lie within $2.0 \mathrm{~min}$. Since the standards cover the MW range of $103\left(\mathrm{C}_{3}\right.$ diacid) to 215 $\left(\mathrm{C}_{11}\right.$ diacid), species with MW between 250 and $400 \mathrm{Da}$ have relatively larger uncertainties in their quantification. Additional standards need to be analyzed in the future to reduce this uncertainty.

[27] For isobaric compounds, various surrogate standards are chosen for the best match in both RT and structures. As an example, sinapic acid (a common compound found in plant cells) is chosen to quantify the $m / z 294$ isobaric peaks eluting before $13.0 \mathrm{~min}$, while suberic acid (a $\mathrm{C}_{8}$ diacid) is chosen to quantify the 294 peaks eluting after $13.0 \mathrm{~min}$. The RT discrepancies between these peaks and the surrogate standards are always less than $0.5 \mathrm{~min}$. Isobaric species with closest RT can be grouped together, and these groups of isobaric species display a rather constant mass distribution pattern in the SE US in June 2004. For example, Figure 9 shows that on 17 and 29 June 2004, the first group of the $\mathrm{m} / \mathrm{z} 294$ isobaric species always has the highest abundance while the second always has the lowest, at all three inland sites. Therefore the summation of these groups is still a good representation of the abundance of all individual isobaric species. With this in mind, we report in Table 1 the mass concentration ( $\mathrm{ng} / \mathrm{m}^{3}$ air) of each detected and quantified species from the quartz filter analyses, without differentiating the isobaric species.

[28] During June 2004, PTFE membrane filters were collected in the SEARCH network with the same sampling protocol as the quartz filters, and they were analyzed by the same LC-MS method. By comparison, the most abundant 


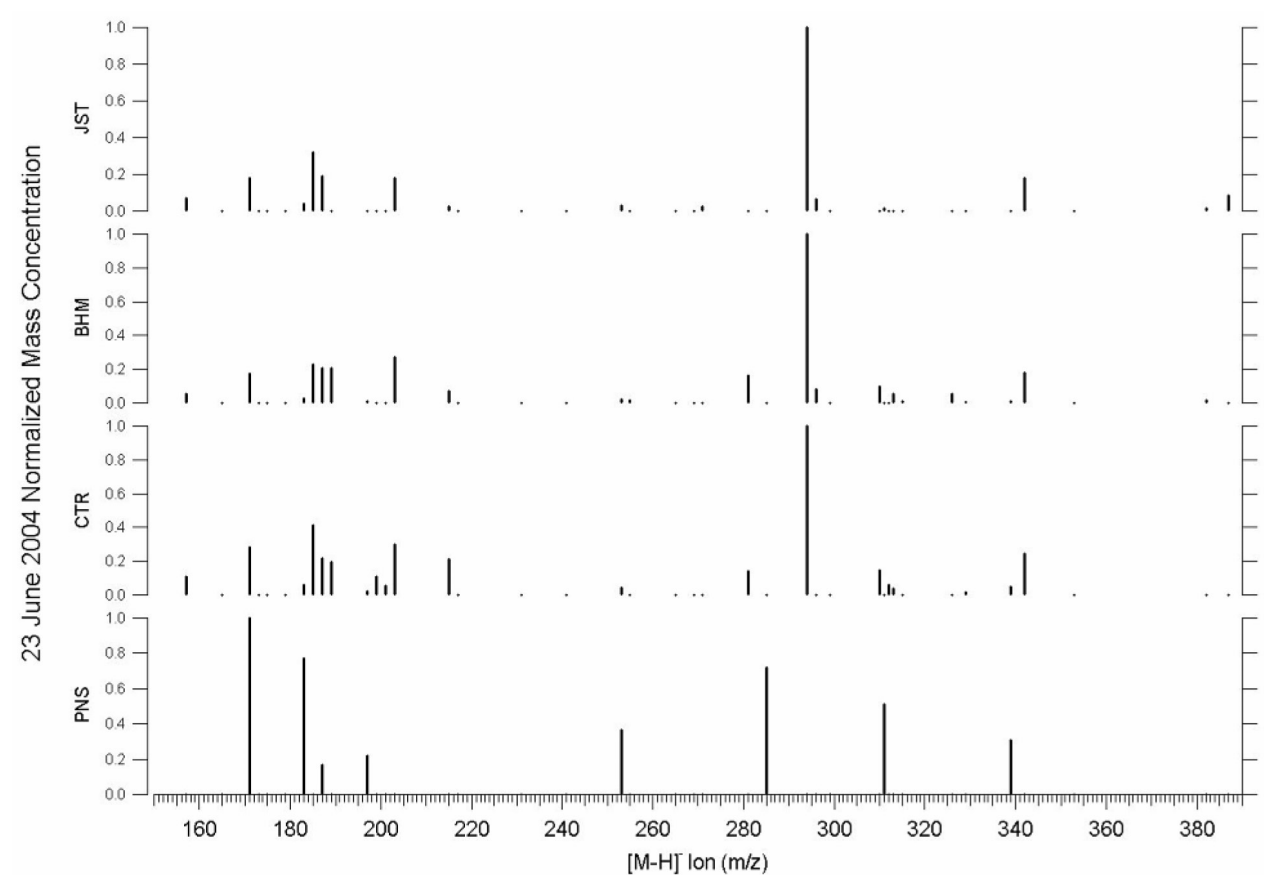

Figure 10. Mass distribution pattern at four sampling sites on 23 June 2004.

species detected on both filters are the same, and the mass distribution pattern of various species in each site is similar. However, the mass concentrations derived from PTFE filters are in general lower, which may be partly due to their generally higher blank filter background. Therefore the type of filters used can have an effect on the measured concentrations of aerosol components, but does not influence the qualitative assessment of aerosol composition. The discussion in this paper is based on quartz filters, which have cleaner blanks. Background signals from blank filters are used to correct for the quantification of field samples.

[29] Some common species are consistently detected as the most abundant species at BHM, CTR, and JST sites, such as the $m / z 171,185,187,203,215,294$ and 342 ions (all molecular ions $[\mathrm{M}-\mathrm{H}]^{-}$). This suggests there are common sources leading to the formation of these species at these continental sites. In comparison, the PNS site has somewhat different aerosol composition, such as the absence of $m / z 294$ and 342 ions and the relatively higher abundance of 285 and 157 ions on some days. This coastal site may receive emission impacts from different sources in comparison to the inland sites. To better understand this difference, we will examine next the spatial and temporal distribution patterns of the detected $\mathrm{PM}_{2.5}$ components in detail.

\subsection{Mass Distribution and Regional Signature of Detected Organic Species}

[30] As will be discussed in section 3.5, these detected species comprise only a modest fraction (on average $7.2 \%$ for the three inland sites and $1.1 \%$ for the PNS site) of the total organic matter in the $\mathrm{PM}_{2.5}$ in the SE US. Since they are relatively polar species, they may be important in determining the hygroscopicity and $\mathrm{CCN}$ activation behavior of aerosols. In addition, as will be discussed in section 3.4, some of these can be tracers for $\mathrm{PM}_{2.5}$ and secondary formation pathways in that region. For each sample, we normalize the mass concentrations of all species to that of the most abundant species. Such a plot displays the mass distribution pattern of the detected $\mathrm{PM}_{2.5}$ components.

[31] For each sampling date, mass distributions at the four sites are plotted in one figure to examine the spatial variation of the detected species across the SE US. As an example, Figure 10 shows the spatial mass distribution pattern on 23 June 2004. Except for the PNS site, the $\mathrm{m} / \mathrm{z} 171,185,187,203,294$ and 342 ions (all [M-H] ${ }^{-}$) are the dominant species, and the relative abundance of these species is rather consistent on this day. In fact, on each sampling day, there is a rather consistent mass distribution pattern across the JST, BHM and CTR sites. And on all four days, the dominant species remain the same with rather modest variation in their relative abundance from day to day (see another example in auxiliary Figure S2). This regional signature of organic matter in $\mathrm{PM}_{2.5}$ strongly suggests that there are common sources in the inland region of the SE US.

[32] Analyses of back trajectories generated using the National Oceanic and Atmospheric Administration (NOAA) HYbrid Single-Particle Lagrangian Integrated Trajectory (HYSPLIT) Model, version 4.7 (http://www.arl.noaa.gov/ ready/hysplit4.html) reveal that meteorological conditions from 14 to 29 June in 2004 are generally similar at the three inland sites. As an illustration, Figure 11 shows the 3-day back trajectories for 23 June while those of the other 3 days are shown in auxiliary Figures S3, S4 and S5. BHM and CTR sites have essentially the same back trajectories due to their close proximity. On 14 June the trajectories originated mostly from the southeast near the vicinity of Florida panhandle. On 17 June the trajectories extended from the South across southern Louisiana, Mississippi, Alabama and the Florida panhandle (depending on start time and trajectory height during the 24-hour period). On 23 and 29 June the trajectories originated mainly from the southwest crossing eastern Texas, Mississippi and Alabama. In addition, the JST site had trajectories very similar to BHM and CTR sites 


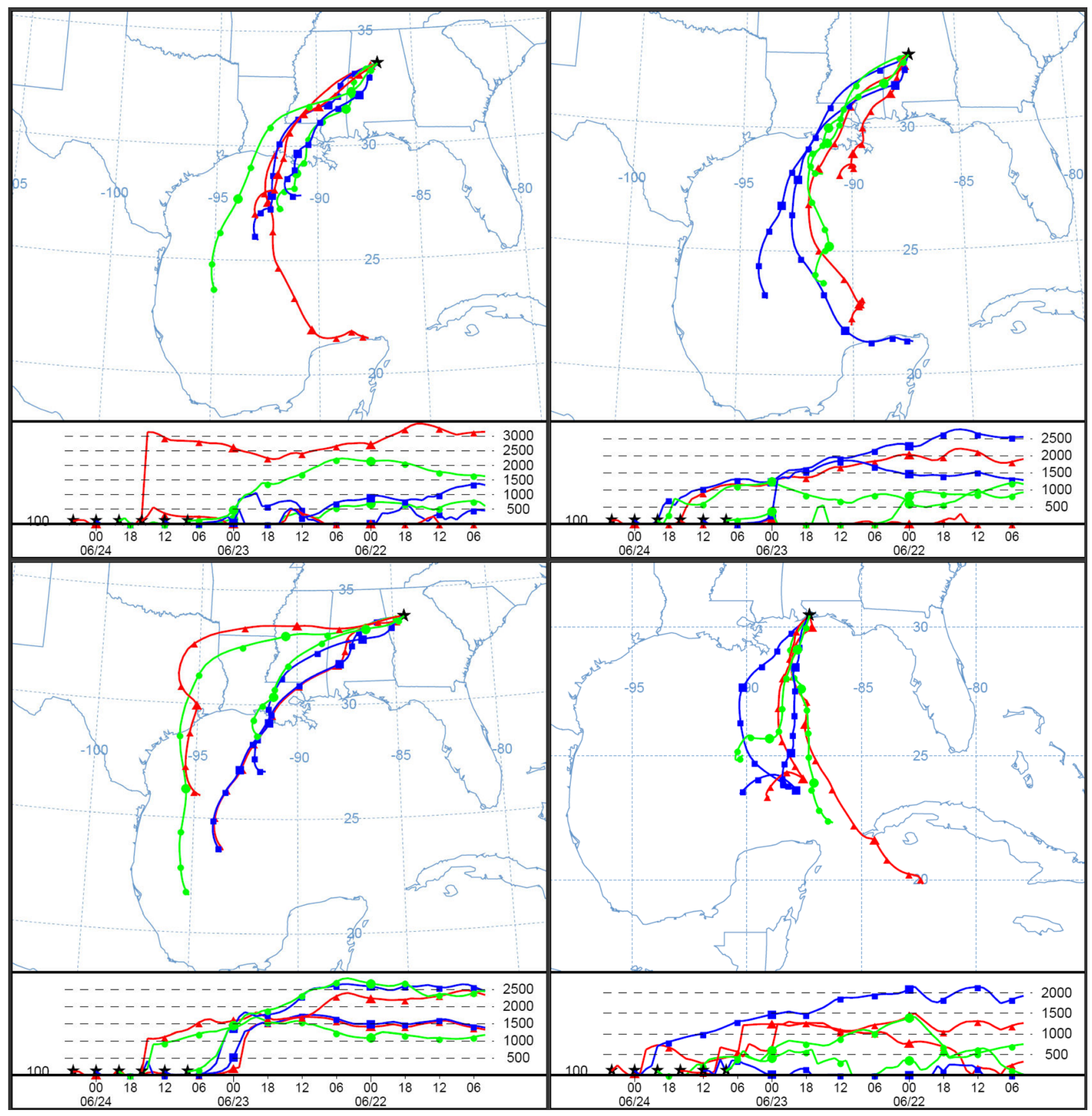

Figure 11. Three-day back trajectories on 23 June 2004 (clockwise from top left: BHM, CTR, PNS, and JST). The start height was $100 \mathrm{~m}$. Back trajectories started every 4 hours starting at 1200 am EDT.

on 17 and 23 June, while on 14 and 29 June the back trajectories extended from SE Georgia/NE Florida and mixed sources, respectively. Owing to the presence of several coal-fired powered plants, extensive industrial activity in the Gulf Coast, and large metropolitan areas near (Atlanta, Birmingham) and upwind of the sampling sites (Houston, New Orleans-Baton Rouge, Mobile-Pensacola, Jacksonville, Orlando, and Tampa-St. Petersburg), all sets of trajectories transported $\mathrm{NO}_{\mathrm{x}}$ and $\mathrm{SO}_{2}$ within a rich, biogenic VOC background en route. All over the SE US, there is a ubiquitous distribution of lush vegetation, much of which emits terpenes abundantly year round, but with higher emissions rates during the summer. Such similar source gases and meteorological conditions would then provide a regional environment for common in situ reac- tions, in particular terpene oxidations, with possible participation of $\mathrm{NO}_{\mathrm{x}}$ and $\mathrm{SO}_{2}$ as proposed earlier based on chemical analysis. Owing to the lower volatility of the products, secondary organic aerosols can subsequently form and contribute significantly to the regional aerosol mass loading. The remarkable similarity in the organic aerosol composition of $\mathrm{PM}_{2.5}$ at JST, BHM and CTR sites on 17 and 23 June (auxiliary Figure S2 and Figure 10) strongly supports such a scheme of SOA formation in the inland region.

[33] In comparison, PNS is a coastal site on the northern edge of the Gulf of Mexico, and experienced mainly southerly flows from the Gulf in June 2004 according to back trajectory analyses (Figure 11 and auxiliary Figures S3, S4 and S5). Such flows and geographical location probably 


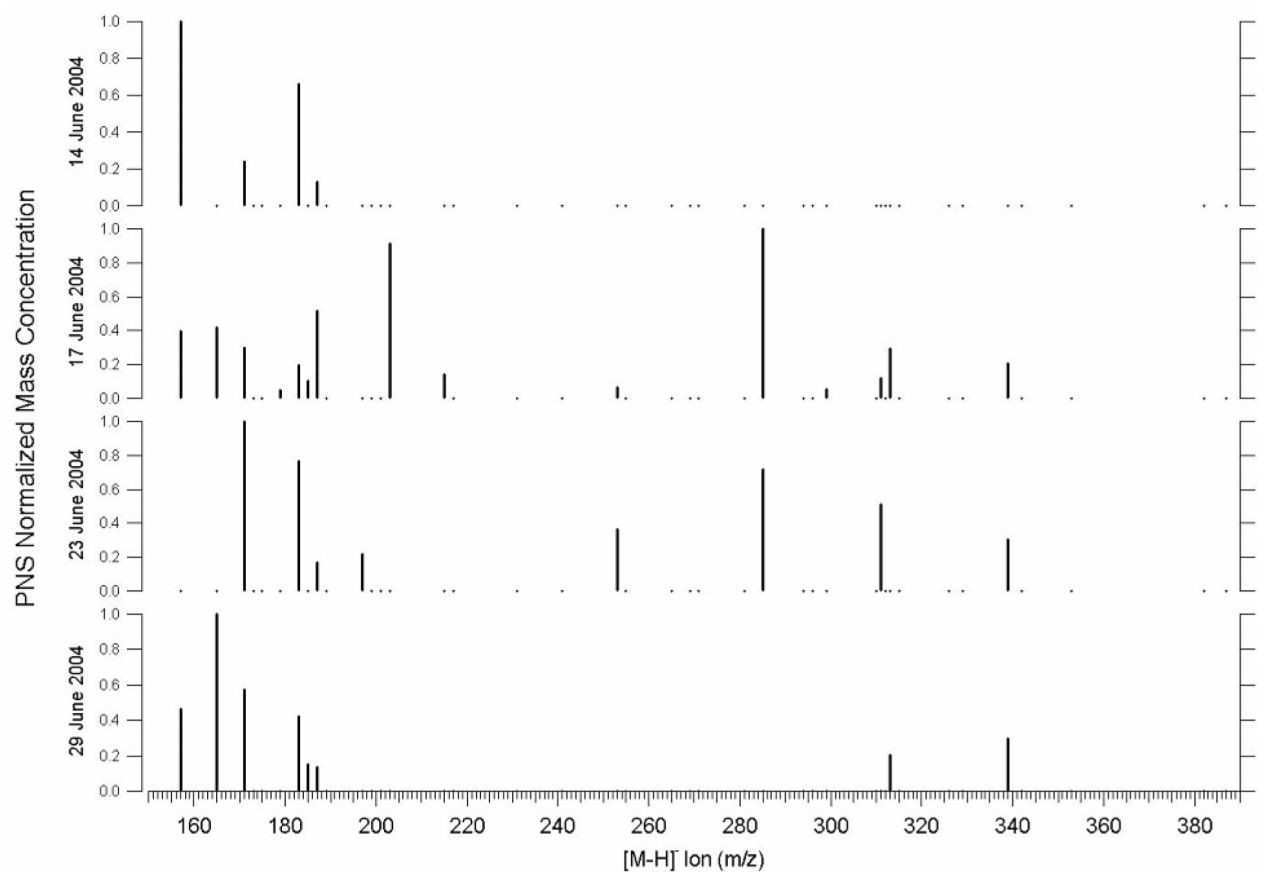

Figure 12. Mass distribution pattern on four sampling dates at the PNS site.

led to a decreased concentration of terpenes, making it difficult to form the $\mathrm{m} / \mathrm{z} 294$ ion according to our proposed scheme. In addition, such trajectories would make it difficult to provide enough $\mathrm{SO}_{2}$ en route to the sampling site, again suppressing formation of the $\mathrm{m} / \mathrm{z} 294$ ion. Indeed, this species is never identified in the $\mathrm{PM}_{2.5}$ samples at the PNS site, despite emissions of $\mathrm{NO}_{\mathrm{x}}$ and VOC from local transportation sources. Furthermore, back trajectory analyses reveal distinctly different flows on the four sampling dates, i.e., south-southeasterly flow (originating from the vicinity of Cuba) on 14 June, southeasterly flow (originating from middle Florida) on 17 June, southerly flow (from the vicinity of the Yucatan peninsula in Mexico) on 23 June and stagnant south-southeasterly flow on 29 June. If primary, oceanic sources from the Gulf of Mexico were mainly responsible for $\mathrm{PM}_{2.5}$ at the PNS site, then the aerosol composition would have been similar on all four sampling days. However, this is not the case as seen in Figure 12, where the temporal mass distribution pattern at the PNS site is shown. Neither the most abundant species nor their relative abundance displays a consistent pattern. Therefore either other primary sources or secondary processes during the onshore transport appear to have played a role in influencing the $\mathrm{PM}_{2.5}$ composition at this site in June 2004. Among the four sites, the fraction of identified organics in the total $\mathrm{PM}_{2.5}$ organic matter is the lowest at the PNS site $(0.4-1.7 \%)$. In contrast, 5.8-9.1\% (temporal average for each site) of the total organic matter is identified for the other three sites (Table 1), and the temporal distribution pattern for identified species is, again, rather consistent. An example is shown in Figure 13, where only the 29 June case for the BHM site has somewhat different mass distribution. Interestingly, the identified organic fraction in this case is $2.5 \%$, the lowest of all 12 continental samples. There was primarily flow from the southwest in near stagnant conditions mainly over Mississippi and Louisiana but originating from the center of the Gulf of Mexico. This unique meteorological condition, and consequently the aerosol evolution, was likely responsible for the above observations on 29 June. On other days, however, it appears there is a rather stable June signature of the $\mathrm{PM}_{2.5}$ composition at the BHM site. This is also true for the JST and CTR sites.

[34] The mass distribution pattern shown in Figure 10 from the LC-MS analyses resembles a typical mass spectrum, and can be readily compared with the corresponding ion trap mass spectrum, such as the Figure 8 for the BHM sample collected on 23 June. These two spectra show very similar distribution patterns, detecting ions with $\mathrm{m} / \mathrm{z} 171$,

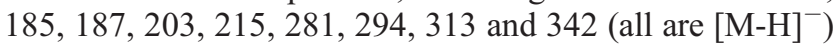
as the most abundant species. In other samples, such a comparison also yields similar mass distribution patterns, which indicates the consistency of our measurements and confirms the stable regional characteristics of fine particulate organic matter, especially the polar components being targeted here.

\subsection{Sources and Evolution of Detected Organic Species: Correlation Study}

[35] Another approach to understanding the sources of detected organic species in $\mathrm{PM}_{2.5}$ is to examine the correlation between the abundances of these species. To account for the variation in the total organic mass in each sample, the mass concentration of an organic species is normalized by its corresponding total organic mass. Standard leastsquare linear regressions are then carried out between the normalized mass concentrations of two species, and their correlation during SEARCH 2004, if statistically significant, indicates that they likely share common sources and evolution pathways. Such a correlation analysis is also carried out between the mass concentration of a species and other field variables, such as the degree of neutraliza- 


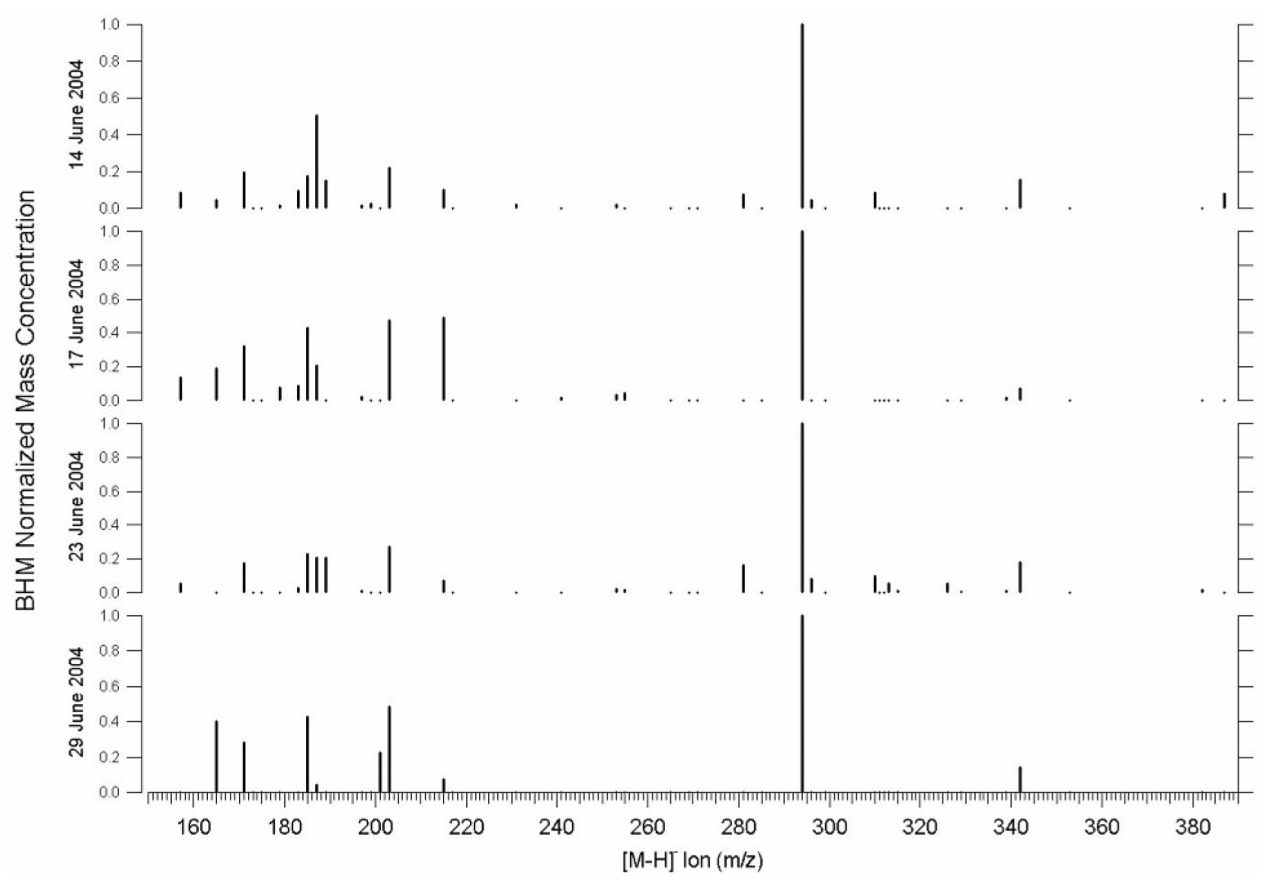

Figure 13. Mass distribution pattern on four sampling dates at the BHM site.

tion of the particle (a proxy for particle acidity), mean wind speed, relative humidity and temperature to examine other possible effects on aerosol composition.

[36] As discussed earlier, the $m / z 171,183$ and 185 ions are very likely oxidation products of monoterpenes. Their ubiquitous presence in $\mathrm{PM}_{2.5}$ in the SE US in June 2004 strongly suggests that terpene oxidation is a common secondary source for some $\mathrm{PM}_{2.5}$ components, and these three species can serve as tracers for such a biogenic source. We next examine the correlation between the abundances of other species and these tracer species. In the MW range of 150-250 Da, 197, 203 and 215 ions are also commonly detected. Table 2 shows the calculated coefficients of determination $\left(\mathrm{R}^{2}\right)$ values of the normalized mass concentrations of these species with that of $m / z 171$ and 185 ions, respectively. Also listed are the numbers of data pairs with positive detection and the corresponding threshold $\mathrm{R}^{2}$ values to be statistically significant at the 5\% level. Although alternative methods exist that can better determine the correlation of data sets taking into account measurement uncertainty, given the small number of data pairs, the significance of correlation is simply estimated as $\left(\mathrm{R}^{2}\right.$ calculated $-\mathrm{R}_{\text {threshold }}^{2} /\left(1-\mathrm{R}^{2}\right.$ threshold $)$, which varies from 0 (at the threshold for a significant correlation at the 5\% level) to
$100 \%$ (at a perfect correlation). It can be seen that the $\mathrm{m} / \mathrm{z}$ 197, 203 and 215 ions all have good or strong correlations with the $m / z \quad 171$ and 185 ions. Therefore terpene oxidation appears to be also a major source for the 197, 203 and 215 ions. Indeed, their ion trap MS/MS spectra are consistent with the structures we propose in Figure 3, and their formation can be explained by known reaction mechanisms, in some cases with peracid $(\mathrm{RC}(\mathrm{O}) \mathrm{OOH})$ as an end product [e.g., Yu et al., 1999; Winterhalter et al., 2003; Aschmann et al., 2003].

[37] Whether terpene oxidation can also be a source for higher-MW $\mathrm{PM}_{2.5}$ species (MW above $250 \mathrm{Da}$ ) can be assessed by similar correlation analysis. We first examine the most abundant species in the three inland sites, the $\mathrm{m} / \mathrm{z} 294$ ion. Using $\mathrm{m} / \mathrm{z} 171,185,187,197,203$ and 215 ions as tracers, it can be seen that the $\mathrm{m} / \mathrm{z} 294$ ion is well correlated with all these species, as shown in Table 3. Combined with the earlier analyses of possible structures and reaction pathways of the $m / z 294$ ion in sections 3.1 and 3.3 , there is strong evidence that terpene oxidation is probably a major source for the $m / z 294$ ion, at least for some of its isobaric species. In addition, some other frequently detected species, such as 253, 339 and 342 ions, are also reasonably well correlated with the tracer species,

Table 2. Correlation of Mass Concentrations of the $m / z$ 197, 203, and 215 Ions With Those of the $m / z 171$ and 185 Ions

\begin{tabular}{|c|c|c|c|c|}
\hline$[\mathrm{M}-\mathrm{H}]$-of $\mathrm{PM}_{2.5}$ Species & $\begin{array}{c}\mathrm{R}^{2} \text { With the Normalized } \\
\text { Mass Concentration of Given Ion }\end{array}$ & Number of Data Pairs & Threshold of $\mathrm{R}^{2}$ Value & $\begin{array}{l}\text { Significance of } \\
\text { Correlation, \% } \\
\end{array}$ \\
\hline \multicolumn{5}{|c|}{171 Ion } \\
\hline 197 & 0.71 & 8 & 0.50 & 43 \\
\hline 203 & 0.80 & 13 & 0.30 & 71 \\
\hline 215 & 0.78 & 13 & 0.30 & 68 \\
\hline \multicolumn{5}{|c|}{185 Ion } \\
\hline 197 & 0.63 & 7 & 0.56 & 16 \\
\hline 215 & 0.50 & 13 & 0.30 & 29 \\
\hline
\end{tabular}


Table 3. Correlation of Mass Concentrations of the $\mathrm{m} / \mathrm{z} 171 \mathrm{Ion}, 185 \mathrm{~m} / \mathrm{z}$ Ion, One of $\mathrm{m} / \mathrm{z} 187$ Isobars, $\mathrm{m} / \mathrm{z} 197$ Ion, $\mathrm{m} / \mathrm{z} 203 \mathrm{Ion}$, and One of $m / z 215$ Isobars With That of the $m / z 294$ Ion

\begin{tabular}{lcccc}
\hline [M-H]-of $\mathrm{PM}_{2.5}$ Species & $\begin{array}{c}\mathrm{R}^{2} \text { With the Normalized Mass } \\
\text { Concentration of the } m / z \text { 294 Ion }\end{array}$ & $\begin{array}{c}\text { Number of } \\
\text { Data Pairs }\end{array}$ & $\begin{array}{c}\text { Threshold } \\
\mathrm{R}^{2} \text { Values }\end{array}$ & $\begin{array}{c}\text { Significance of } \\
\text { Correlation, \% }\end{array}$ \\
\hline 171 & 0.60 & 12 & 0.34 & 40 \\
185 & 0.49 & 12 & 0.34 & 23 \\
$187 \mathrm{~A}$ & 0.70 & 8 & 0.50 & 7 \\
197 & 0.59 & 7 & 0.56 & 15 \\
203 & 0.44 & 8 & 0.34 & 38 \\
$215 \mathrm{~B}$ & 0.69 & & 0.50 & 38 \\
\hline
\end{tabular}

suggesting a biogenic, secondary source as well. Possible evolution pathways can be readily gleaned from the recent laboratory chamber experiments discussed in section 3.1. Not only is the $\mathrm{m} / \mathrm{z} 294$ ion present in the SOA from $\alpha-$ pinene photooxidation (with the participation of $\mathrm{NO}_{\mathrm{x}}, \mathrm{SO}_{2}$ and sometimes other VOC precursors), but the $\mathrm{m} / \mathrm{z} 171$, 183, 185, 197, 203 and 215 ions are also present. They commonly have isobaric species, some of which elute the LC column at retention times similar to the same-mass $\mathrm{PM}_{2.5}$ species in SEARCH. The upfront CID mass spectra of some of these species are also similar to their counterparts in the SEARCH samples, suggesting that $\alpha$-pinene photooxidation is a possible pathway. Gas-phase data such as $\mathrm{O}_{3}, \mathrm{SO}_{2}$ and $\mathrm{NO}_{\mathrm{x}}$ mixing ratios are needed to fully examine possible evolution pathways of the detected species in $\mathrm{PM}_{2.5}$. Currently, these data are not yet available.

[38] Particle acidity (assessed by the degree of neutralization calculated as the mole-equivalent ratio of ammonium to sulfate and nitrate) is found to have no correlation with any detected species or other field variables obtained in the SEARCH network in June 2004. As discussed in section 3.1, in the MW range 150-400 Da, oligomers do not appear to be present in $\mathrm{PM}_{2.5}$ in the SE US. Therefore acid catalysis and subsequent oligomer formation does not appear to be a main pathway to form major $\mathrm{PM}_{2.5}$ components in this MW range. Rather, oxidation of monoterpenes and sesquiterpenes can readily lead to many of the polar species in $\mathrm{PM}_{2.5}$. Although possibility exists that some species with MW above $400 \mathrm{Da}$ are oligomeric in nature, this is less likely than a direct extrapolation from recent chamber experiments [Kalberer et al., 2004; Tolocka et al., 2004; Gao et al., 2004b] would suggest. The polar nature of the detected species in $\mathrm{PM}_{2.5}$ in the SE US suggests oxidation is an important pathway to form aerosol components, and these oxidation processes, both ozonolysis and photooxidation, can occur continuously in the atmosphere. Albeit unknown at this moment, it is also conceivable that oxidation may be one of the pathways to decompose oligomers. Indeed, as shown theoretically by Barsanti and Pankow [2004], most accretion reactions proposed from chamber studies do not appear to be thermodynamically favorable pathways to form oligomers or grow aerosol mass. It is possible that oligomers observed in chamber experiments, the longest of which lasted less than 30 hours [Kalberer et al., 2004], decompose by various pathways at a prolonged aging time with complex atmospheric processing. The average aging time for tropospheric aerosols is roughly a week. Furthermore, the degradation scheme would suggest that smallest oligomers are most likely to survive in the fine aerosols (unless some large oligomers have highly conjugated structures), as they are near the end of degradation pathways. However, our observations reveal a lack of oligomers in the low MW range (150-400 Da). In all, little evidence exists that oligomers comprise a major fraction of $\mathrm{PM}_{2.5}$ in the SE US in the summer. Rather, strong evidence exists that oxidation is a main pathway to form polar particulate species there. Since similar species have been observed in $\mathrm{PM}_{2.5}$ elsewhere [Gelencser et al., 2000, 2002; Krivacsy et al., 2001; Kiss et al., 2003], this terpene oxidation scheme is likely a ubiquitous pathway to form major organic species in $\mathrm{PM}_{2.5}$ in rural, continental regions.

[39] Even though a detailed source apportionment study has not been carried out in this work, it is possible to estimate the source contribution of terpene oxidation to $\mathrm{PM}_{2.5}$. Since most of the dominant organic species detected in this work are associated with terpene oxidation, together they can provide a lower-bound estimate of its source contribution to the total organic matter in $\mathrm{PM}_{2.5}$ in the SE US, which is $1.1-9.1 \%$ in June 2004. This is comparable to the source contributions from diesel exhaust (14-30\%), meat cooking $(5-12 \%)$, and gasoline-powered motor vehicle exhaust $(0-10 \%)$ in that region [Zheng et al., 2002]. Since wood smoke makes a much smaller contribution (less than $30 \%$ in July 1999) to $\mathrm{PM}_{2.5}$ organic matter in the summer [Zheng et al., 2002], terpene oxidation ranks among the major regional sources during summer. Indeed, the speculation [Zheng et al., 2002] that the exceptionally high ratio of unexplained $\mathrm{OC}$ to the total $\mathrm{OC}$ in the summer in the SE US is due to SOA formation is supported by this work. By the same approach, at JST, CTR, BHM and PNS sites in June 2004, terpene oxidation contributed to at least $0.2-5.2 \%$ of the total fine particulate mass.

\subsection{Identified and Missing Fractions of Particulate Organic Matter}

[40] Six categories of chemical species in $\mathrm{PM}_{2.5}$ were measured in June 2004 in the SEARCH network by various techniques described by Hansen et al. [2003], i.e., sulfate, nitrate, ammonium, black carbon, organic matter and major metal oxides (including $\mathrm{Al}_{2} \mathrm{O}_{3}, \mathrm{SiO}_{2}, \mathrm{~K}_{2} \mathrm{O}, \mathrm{CaO}, \mathrm{TiO}_{2}$, $\mathrm{Fe}_{2} \mathrm{O}_{3}$ ). Together, they comprise $92.1 \pm 6.9 \%, 95.4 \pm$ $5.8 \%, 86.3 \pm 7.0 \%$ and $78.2 \pm 13.7 \%$ of the total $\mathrm{PM}_{2.5}$ mass at JST, CTR, BHM and PNS sites, respectively. Of the six categories, total organic matter (TOM) mass, estimated from multiplying the measured total organic carbon (TOC) mass by the corresponding $\mathrm{OM} / \mathrm{OC}$ ratio, comprise $36.8 \pm$ $5.6 \%, 42.7 \pm 13.6 \%, 31.8 \pm 2.4 \%$ and $20.5 \pm 8.2 \%$ of the total $\mathrm{PM}_{2.5}$ mass at JST, CTR, BHM and PNS sites, respectively. The OM/OC ratio of 1.6 is used for the three mainly urban sites (JST, BHM and PNS) while the ratio of 2.0 is used for the mostly rural site (CTR), as suggested by recent work [e.g., Krivacsy et al., 2001; Turpin and Lim, 2001; El-Zanan et al., 2005]. This is consistent with the relatively high (MW/carbon mass) ratios of many highly 


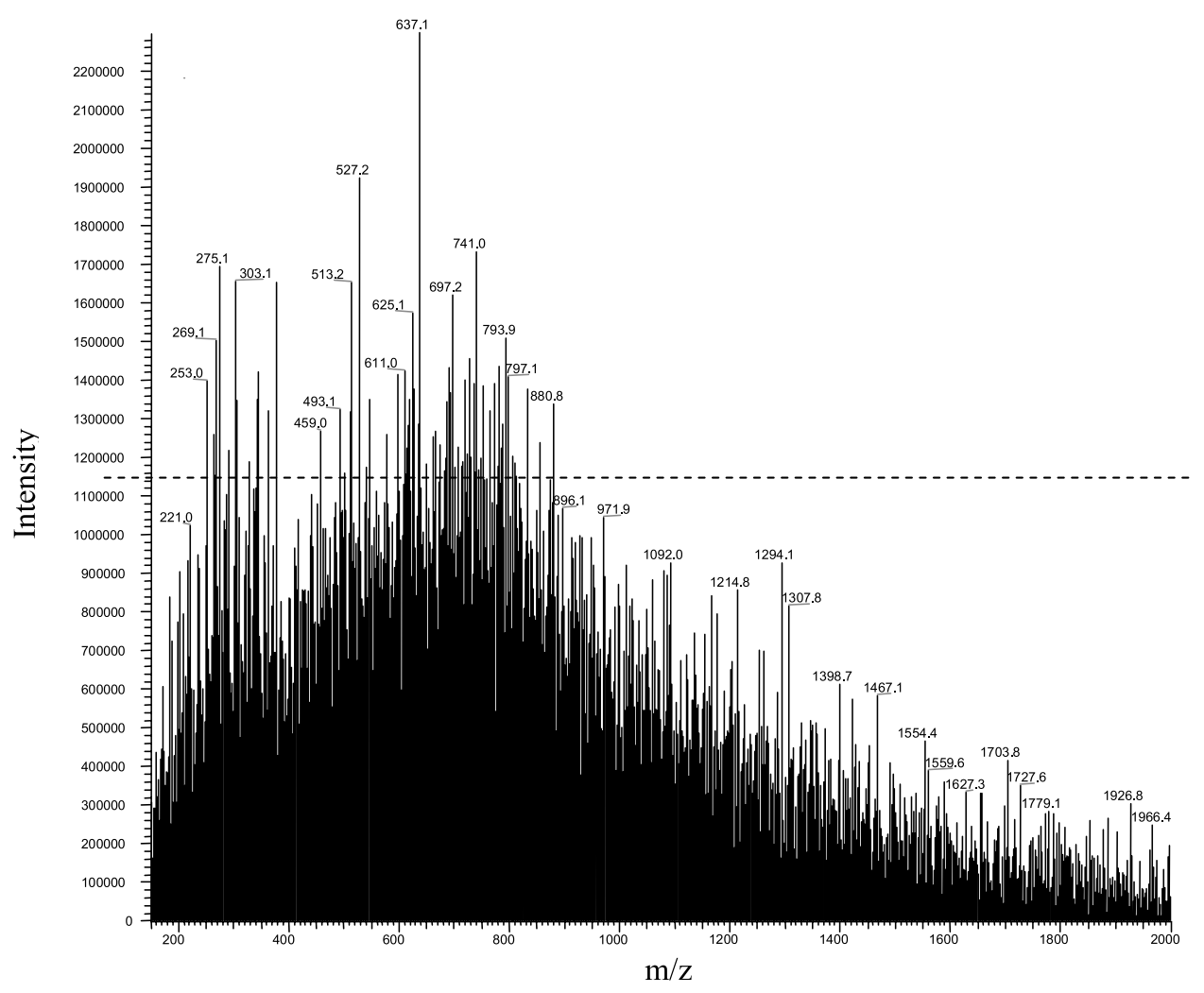

Figure 14. Ion trap MS (positive ion mode) of the extract of the $\mathrm{PM}_{2.5}$ sample collected at the CTR site on 17 June 2004. $\mathrm{PM}_{2.5}$ organic components cover a MW range of 200-850 (after the Na mass is subtracted). The detection limit was determined from field blanks and was $\sim 1,120,000$ as shown by the dashed line.

oxidized species identified in this study (e.g., as high as 2.46 for the $m / z 294$ species). Averaged over the four June samples, the total identified organic species (Table 1) account for $5.8 \%, 9.1 \%, 6.8 \%$ and $1.1 \%$ of the total particulate organic mass at the JST, CTR, BHM and PNS sites, respectively. Questions arise as to the nature of the unidentified organic species in $\mathrm{PM}_{2.5}$.

[41] In the previous composition analysis and source apportionment study of $\mathrm{PM}_{2.5}$ in the SE US, Zheng et al. [2002] detected 107 organic compounds, which accounted for no more than $12 \%$ of the total organic mass in $\mathrm{PM}_{2.5}$. The majority of these detected compounds are rather nonpolar, such as alkanes, cycloalkanes, PAHs, steranes, hopanes, long-chain alkanoic acids and resin acids. This is due to the nature of the GC-MS employed by Zheng et al. [2002], which is amenable primarily to detecting relatively volatile and nonpolar species.

[42] By comparison, LC-MS is used in this work, which is suitable to detecting relatively polar and acidic species. Nonanedioic acid (azelaic acid) is the common diacid that is detected by both Zheng et al. [2002] and this work. Owing to the quantification of all $\mathrm{m} / \mathrm{z} 187$ isobaric species, the mass concentration of the 187 ion is higher in this study (30.63 ng/ $\mathrm{m}^{3}$, on average, for JST, BHM and CTR sites) than in Zheng et al. [2002] $\left(6.28 \mathrm{ng} / \mathrm{m}^{3}\right)$. Interestingly, azelaic acid $\left(\mathrm{C}_{9}\right)$ is a well-known secondary oxidation product of unsaturated fatty acids [Stephanou and Stratigakis, 1993], again suggesting a secondary pathway for organic aerosols in the SE US. Smaller diacids $\left(\mathrm{C}_{3}\right.$ to $\mathrm{C}_{8}$ ) were detected by the GC-MS, but they were not detected by the LC-MS, which is probably due to the loss during the water washing step of the SPE technique. The water effluents from SPE of all samples have been archived at $-20^{\circ} \mathrm{C}$, and can be analyzed if necessary. However, these diacids often have low occurrences [Yu et al., 2005] in the SE US and are rarely more than $7 \mathrm{ng} / \mathrm{m}^{3}$ in $\mathrm{PM}_{2.5}$ [Zheng et al., 2002]. In total, they are estimated to comprise only a small fraction of the total organic mass in $\mathrm{PM}_{2.5}$, consistent with observations at other rural continental sites [e.g., Sarvari et al., 1999]. The organic species detected in this work, most of which appear to originate from terpene oxidation, comprise on average $7.2 \%$ of the total organic mass in $\mathrm{PM}_{2.5}$ at the three inland sites. Combining with the speciation results by Zheng et al. [2002], about $20 \%$ of the $\mathrm{PM}_{2.5}$ organic matter has been quantitatively identified.

[43] Whereas the chemical nature of the unidentified organic species remains elusive, it is possible to estimate the MW range of these species. Under the positive ion mode in the ion trap MS, compounds with a broad range of polarity can be detected as their $\mathrm{Na}^{+}$adducts, as demonstrated by Gao et al. [2004b]. Figure 14 shows the ion trap mass spectrum of a $\mathrm{PM}_{2.5}$ sample collected in the CTR site on 17 June 2004, which was extracted with the SPE technique applied. The detection limit is determined from the average and standard deviation of six field blanks with a signal-to-noise ratio of 3 applied. It can be seen that compounds with MW from $\sim 200-850 \mathrm{Da}$ are present in this sample. When SPE is not applied during filter extraction, most species increase in intensity by a factor of 2-3 and species with MW above 1000 are present, which is 
probably due to the adduct formation between charged molecules and neutral ones and/or between metal ions and organic species [Klaus et al., 2000; Leenheer et al., 2001; Plancque et al., 2001]. In the mass spectra where inorganic ions are mostly removed by SPE, such as the one shown in Figure 14, the chance of adduct formation has been minimized. Therefore the MW range of $\mathrm{PM}_{2.5}$ organic matter is estimated to be 200-850 Da in this sample. Other samples are evaluated by the same approach. On average, organic species in $\mathrm{PM}_{2.5}$ in the SE US in June 2004 cover a MW range of 150-800 Da. This is consistent with the recent estimate that the upper MW limit of HULIS in ambient urban aerosols is about 700 [Samburova et al., 2005]. This latter estimate was based on two different analytical techniques, i.e., size exclusion chromatography-UV spectroscopy and LDI MS, providing some independent confirmation of this conclusion.

[44] It can be seen from Figure 14 that compounds with MW above 400 Da actually have higher abundances and number of species than those with MW below 400 Da. If similar response factors are assumed for all detected compounds, those with MW above 400 Da may comprise a major fraction of the total organic matter. This suggests that these relatively high-MW species, whose molecular structures are not yet known, may explain a substantial fraction of unidentified mass in $\mathrm{PM}_{2.5}$. However, it is possible that there is large variation in response factors among species, especially the high-MW ones of which response factors are not well understood. In addition, despite the application of SPE, adduct formation in the ion trap MS is still likely which may have caused a shift in the MW range to larger numbers.

[45] Higher-MW, relatively polar, but unidentified species in $\mathrm{PM}_{2.5}$ have been lumped together and named HULIS owing to a speculation of their origin as the humic (and/or fulvic) acids found in natural waters and soil [e.g., Havers et al., 1998] (and references mentioned above). A primary source for HULIS (i.e., direct release of actual humic matter from the soil or natural waters) appears unlikely, owing to the ubiquitous presence of HULIS in the fine particle size range. It has been hypothesized that HULIS in continental $\mathrm{PM}_{2.5}$ may be produced aloft from precursors emitted directly from terrestrial sources [Gelencser et al., 2002]. However, calculation by the same authors show that this soil flux appears too low (by about 1 order of magnitude) to account for the observed total organic mass in $\mathrm{PM}_{2.5}$. On the other hand, a photochemical secondary source for HULIS has also been speculated [Gelencser et al., 2002; Szidat et al., 2004; Samburova et al., 2005]. To our knowledge, we have presented the first, direct evidence that some HULIS species are probably formed via atmospheric oxidation of VOC precursors. In the SE US, polar species with MW range 150-400 Da in $\mathrm{PM}_{2.5}$ appear to originate from the oxidation of monoterpenes and sesquiterpenes. The ESI-mass spectra of $\mathrm{PM}_{2.5}$ samples in the SE US bear resemblance to those of aerosol and fog water samples in previous studies, such as those of Krivacsy et al. [2001], Kiss et al. [2003] and Cappiello et al. [2003], suggesting that similar HULIS material is present in $\mathrm{PM}_{2.5}$ in different regions. A biogenic, secondary source can be ubiquitous in the background atmosphere in many continental regions, leading to a multitude of relatively polar and acidic species in $\mathrm{PM}_{2.5}$. The wide-ranging biogenic precursors and the complex reaction pathways can result in a series of secondary aerosol compo- nents with extremely similar but not identical structures. This would be consistent with the commonly observed coelution of HULIS species on LC columns and regular mass differences seen in the mass spectra. Of course, the structural similarity between HULIS species in $\mathrm{PM}_{2.5}$ and humic/fulvic acids in natural waters and soil [Martin et al., 1994, 1995; Gelencser et al., 2000; Krivacsy et al., 2001] still indicates a possible link between them, yet direct evidence has yet to be found. Indeed, it is likely that both pathways exist and the resultant ensemble of species is the so-called HULIS in $\mathrm{PM}_{2.5}$. Indirect evidence for this appears to exist, such as the somewhat different chemical nature between the $\mathrm{m} / \mathrm{z} 294$ ion and other species in rural fine aerosols as reported by Kiss et al. [2003]. In addition, it is possible that oligomerization may be another pathway in forming HULIS species in $\mathrm{PM}_{2.5}$, such as those with MW above $400 \mathrm{Da}$ that likely contain highly conjugated monomer units. Novel techniques to unravel the structures of higher-MW species and unambiguously detect oligomers should be pursued. The ability to quantify higher-MW species should also be pursued to achieve a better mass closure of organic matter in $\mathrm{PM}_{2.5}$.

\section{Summary and Conclusions}

[46] Four analytical techniques have been used concurrently to identify and quantify polar organic components in $\mathrm{PM}_{2.5}$ in the SE US. Forty distinct species are detected and together they comprise on average $7.2 \%$ and $1.1 \%$ of the total organic mass in $\mathrm{PM}_{2.5}$ at three inland sites and a coastal site, respectively. These polar, acidic species cover a MW range of 150-400 Da and do not appear to be oligomeric in nature. The mass distribution pattern of these species is rather consistent in the inland region due to the rather uniform sources and meteorology, but this pattern varies in the coastal region owing to the more complex sources and meteorology.

[47] Chemical structure and correlation analyses strongly suggest that most of these polar species originate from terpene oxidation. Structures of some species are proposed on the basis of fragmentation patterns in the MS/MS spectra and known reaction mechanisms. In particular, the most dominant species $\left([\mathrm{M}-\mathrm{H}]^{-}\right.$has $\mathrm{m} / z$ 294) is likely from monoterpene oxidation with the participation of $\mathrm{NO}_{\mathrm{x}}$ and $\mathrm{SO}_{2}$ from anthropogenic sources. It is estimated that terpene oxidation can be an important regional source for the organic fraction of $\mathrm{PM}_{2.5}$ in the SE US, contributing to $1.1-9.1 \%$ of the total organic mass. Proposed oxidation pathways can lead to other polar and possibly higher-MW species in fine aerosols that have up to now evaded detection. There is no direct evidence that oligomerization or particle acidity plays a role in affecting the aged aerosol composition or mass. The sufficient aging in the atmosphere may decompose oligomers (if formed initially), which is yet difficult to simulate in laboratory chamber studies. Nevertheless, in the MW range of 400-800 Da where organic species are detected but unidentified, it cannot be precluded that oligomers may be present and could comprise a fraction of the total particulate organic mass.

[48] In rural areas in other parts of the world, terpene oxidation can also be an important regional source, leading to the formation of secondary organic aerosols. Indeed, a biogenic, secondary source is likely ubiquitous for $\mathrm{PM}_{2.5}$ in 
the background atmosphere in continental regions, forming some of the so-called HULIS species. We provide the first direct evidence for this hypothesis. The validity and relative importance of this source and the hypothetical natural source (terrestrial and/or aquatic humic/fulvic acids) for HULIS in fine aerosols await further investigation.

[49] Acknowledgments. This work was supported by the Electric Power Research Institute (EPRI). Jason Surratt was supported by an EPA Science to Achieve Results (STAR) Fellowship. We thank D. Alan Hansen of Electric Power Research Institute and John Jansen of Southern Company for helpful discussions. We also thank Mei Zheng at the Georgia Institute of Technology for preparing and shipping the quartz filter samples and John Greaves at the University of California, Irvine, for the accurate mass measurements on the ESI-TOF instrument.

\section{References}

Aschmann, S. M., E. C. Tuazon, J. Arey, and R. Atkinson (2003), Products of the gas-phase reaction of $\mathrm{O}_{3}$ with cyclohexene, J. Phys. Chem. A, 107, $2247-2255$

Baltensperger, U., et al. (2005), Secondary organic aerosols from anthropogenic and biogenic precursors, Faraday Disc., 130, 265-278.

Barsanti, K., and J. Pankow (2004), Thermodynamics of the formation of atmospheric organic particulate matter by accretion reactions: Part 1 . Aldehydes and ketones, Atmos. Environ., 38, 4371-4382.

Buxton, G. V., G. A. Salmon, and J. E. Williams (2000), The reactivity of biogenic monoterpenes towards $\mathrm{OH}$ center dot and SO4-center dot radicals in de-oxygenated acidic solution, J. Atmos. Chem., 36, 111-134.

Cappiello, A., E. De Simoni, C. Fiorucci, F. Mangani, P. Palma, H. Trufelli, S. Decesari, M. C. Facchini, and S. Fuzzi (2003), Molecular characterization of the water-soluble organic compounds in fogwater by ESIMS/ MS, Environ. Sci. Technol., 37, 1229-1240.

Choi, M., and C. Chan (2002), The effects of organic species on the hygroscopic behaviors of inorganic aerosols, Environ. Sci. Technol., 36, $2422-$ 2428.

Cruz, C., and S. Pandis (1998), The effect of organic coatings on the cloud condensation nuclei activation of inorganic atmospheric aerosol, J. Geophys. Res., 103, 13,111-13,123.

Cruz, C., and S. Pandis (2000), Deliquescence and hygroscopic growth of mixed inorganic-organic atmospheric aerosol, Environ. Sci. Technol., 34, 4313-4319.

El-Zanan, H. S., D. H. Lowenthal, B. Zielinska, J. C. Chow, and N. Kumar (2005), Determination of the organic aerosol mass to organic carbon ratio in IMPROVE samples, Chemosphere, 60, 485-496.

Gao, S., M. Keywood, N. L. Ng, J. Surratt, V. Varutbangkul, R. Bahreini, R. C. Flagan, and J. H. Seinfeld (2004a), Low-molecular-weight and oligomeric components in secondary organic aerosol from the ozonolysis of cycloalkenes and alpha-pinene, J. Phys. Chem. A, 108, 10,147-10,164.

Gao, S., et al. (2004b), Particle phase acidity and oligomer formation in secondary organic aerosol, Environ. Sci. Technol., 38, 6582-6589.

Gelencser, A., T. Meszaros, M. Blazso, G. Kiss, Z. Krivacsy, A. Molnar, and E. Meszaros (2000), Structural characterisation of organic matter in fine tropospheric aerosol by pyrolysis-gas chromatography-mass spectrometry, J. Atmos. Chem., 37, 173-183.

Gelencser, A., A. Hoffer, Z. Krivacsy, G. Kiss, A. Molnar, and E. Meszaros (2002), On the possible origin of humic matter in fine continental aerosol, J. Geophys. Res., 107(D12), 4137, doi:10.1029/2001JD001299.

Glasius, M., M. Lahaniati, A. Calogirou, D. Di Bella, N. R. Jensen, J. Hjorth, D. Kotzias, and B. R. Larsen (2000), Carboxylic acids in secondary aerosols from oxidation of cyclic monoterpenes by ozone, Environ. Sci. Technol., 34, 1001-1010.

Hansen, D., E. S. Edgerton, B. E. Hartsell, J. J. Jansen, N. Kandasamy, G. M. Hidy, and C. L. Blanchard (2003), The southeastern aerosol research and characterization study: Part 1, Overview, J. Air Waste Manage., 53, 1460-1471.

Havers, N., P. Burba, J. Lambert, and D. Klockow (1998), Spectroscopic characterization of humic-like substances in airborne particulate matter, J. Atmos. Chem., 29, 45-54.

Kalberer, M., et al. (2004), Identification of polymers as major components of atmospheric organic aerosols, Science, 303, 1659-1662.

Kavouras, I. G., N. Mihalopoulos, and E. G. Stephanou (1999), Formation and gas/particle partitioning of monoterpenes photooxidation products over forests, Geophys. Res. Lett., 26, 55-58.

Kiss, G., B. Varga, A. Gelencser, Z. Krivacsy, A. Molnar, T. Alsberg, L. Persson, H. C. Hansson, and M. C. Facchini (2001), Characterisation of polar organic compounds in fog water, Atmos. Environ., 35, $2193-$ 2200 .
Kiss, G., E. Tombacz, B. Varga, T. Alsberg, and L. Persson (2003), Estimation of the average molecular weight of humic-like substances isolated from fine atmospheric aerosol, Atmos. Environ., 37, 3783-3794.

Klaus, U., T. Pfeifer, and M. Spiteller (2000), APCI-MS/MS: A powerful tool for the analysis of bound residues resulting from the interaction of pesticides with DOM and humic substances, Environ. Sci. Technol., 34, 3514-3520.

Krivacsy, Z., et al. (2001), Study on the chemical character of water soluble organic compounds in fine atmospheric aerosol at the Jungfraujoch, J. Atmos. Chem., 39, 235-259.

Leenheer, J. A., C. E. Rostad, P. M. Gates, E. T. Furlong, and I. Ferrer (2001), Molecular resolution and fragmentation of fulvic acid by electrospray ionization/multistage tandem mass spectrometry, Anal. Chem., 73 , $1461-1471$

Lim, H. J., and B. J. Turpin (2002), Origins of primary and secondary organic aerosol in Atlanta: Results of time-resolved measurements during the Atlanta supersite experiment, Environ. Sci. Technol., 36, 4489-4496.

Martin, F., F. J. Gonzalezvila, J. C. Delrio, and T. Verdejo (1994), Pyrolysis derivatization of humic substances: 1. Pyrolysis of fulvic-acids in the presence of tetramethylammonium hydroxide, J. Anal. Appl. Pyrol., 28, $71-80$.

Martin, F., J. C. Delrio, F. J. Gonzalezvila, and T. Verdejo (1995), Pyrolysis derivatization of humic substances: 2 . Pyrolysis of soil humic acid in the presence of tetramethylammonium hydroxide, J. Anal. Appl. Pyrol., 31, $75-83$.

Pio, C. A., C. A. Alves, and A. C. Duarte (2001), Organic components of aerosols in a forested area of central Greece, Atmos. Environ., 35, 389-401.

Plancque, G., B. Amekraz, V. Moulin, P. Toulhoat, and C. Moulin (2001), Molecular structure of fulvic acids by electrospray with quadrupole timeof-flight mass spectrometry, Rapid Commun. Mass. Spectrom., 15, 827835 .

Samburova, V., M. Kalberer, and R. Zenobi (2005), Characterization of high molecular weight compounds in urban atmospheric particles, Atmos. Chem. Phys. Disc., 5, 437-454.

Sarvari, Z., Z. Krivacsy, U. Baltensperger, S. Nyeki, E. Weingartner, S. Wessel, and S. G. Jennings (1999), Low-molecular weight carboxylic acids in atmospheric aerosol at different European sites, J. Aerosol Sci., 30, S261-S262.

Stephanou, E. G., and N. Stratigakis (1993), Oxocarboxylic and alpha, omega-dicarboxylic acids-Photooxidation products of biogenic unsaturated fatty-acids present in urban aerosols, Environ. Sci. Technol., 27, $1403-1407$

Szidat, S., et al. (2004), Source apportionment of aerosols by C-14 measurements in different carbonaceous particle fractions, Radiocarbon, 46, $475-484$

Tolocka, M. P., M. Jang, J. M. Ginter, F. J. Cox, R. M. Kamens, and M. V. Johnston (2004), Formation of oligomers in secondary organic aerosol, Environ. Sci. Technol., 38, 1428-1434.

Turpin, B. J., and H. J. Lim (2001), Species contributions to PM2.5 mass concentrations: Revisiting common assumptions for estimating organic mass, Aerosol Sci. Technol., 35, 602-610.

Turpin, B. J., J. J. Huntzicker, and S. V. Hering (1994), Investigation of organic aerosol sampling artifacts in the Los-Angeles basin, Atmos. Environ., 28, 3061-3071.

Winterhalter, R., R. Van Dingenen, B. R. Larsen, N. R. Jensen, and J. Hjorth (2003), LC-MS analysis of aerosol particles from the oxidation of a-pinene by ozone and OH-radicals, Atmos. Chem. Phys. Disc., 3, 1-39.

Yu, J. Z., D. R. Cocker, R. J. Griffin, R. C. Flagan, and J. H. Seinfeld (1999), Gas-phase ozone oxidation of monoterpenes: Gaseous and particulate products, J. Atmos. Chem., 34, 207-258.

Yu, L. E., M. L. Shulman, R. Kopperud, and L. M. Hildemann (2005), Characterization of organic compounds collected during Southeastern Aerosol and Visibility Study: Water-soluble organic species, Environ. Sci. Technol., 39, 707-715.

Zheng, M., G. R. Cass, J. J. Schauer, and E. S. Edgerton (2002), Source apportionment of PM2.5 in the southeastern United States using solventextractable organic compounds as tracers, Environ. Sci. Technol., 36, $2361-2371$.

E. S. Edgerton, Atmospheric Research and Analysis, Inc., Cary, NC 27513, USA.

S. Gao and J. H. Seinfeld, Departments of Environmental Science and Engineering and Chemical Engineering, California Institute of Technology, 1200 East California Boulevard, MC 210-41, Pasadena, CA 91125, USA. (seinfeld@caltech.edu)

E. M. Knipping, Electric Power Research Institute, Palo Alto, CA 94304 USA.

M. Shahgholi and J. D. Surratt, Department of Chemistry, California Institute of Technology, Pasadena, CA 91125, USA. 